\title{
Comprehensive-Double Slit Experiments Violating Wave Description and Trajectory Description ---Exploring Mystery of Double Slit Experiments
}

This paper was downloaded from TechRxiv (https://www.techrxiv.org).

\section{LICENSE}

CC BY 4.0

SUBMISSION DATE / POSTED DATE

$10-02-2022$ / 16-02-2022

\section{CITATION}

peng, hui (2022): Comprehensive-Double Slit Experiments Violating Wave Description and Trajectory Description ---Exploring Mystery of Double Slit Experiments. TechRxiv. Preprint.

https://doi.org/10.36227/techrxiv.19158329.v1

DOI

10.36227/techrxiv.19158329.v1 


\title{
Comprehensive-Double Slit Experiments Violating Wave Description and Trajectory Description ---Exploring Mystery of Double Slit Experiments
}

\author{
Hui Peng* \\ *davidpeng1749@gmail.com
}

\begin{abstract}
Young's double slit experiments, which represent the mystery of quantum mechanics (Feynman), have been described by optics, electromagnetic wave theory, quantum wave theory and Bohm's trajectory theory. The double slit has the varieties of the applications. To test the description theories, we perform the comprehensive double slit experiments, which show that (1) before passing through the double slit/cross-double slit and before landing on the screen, the laser light behaves as particles, not as waves; (2) the experiments challenge the computer simulation of the trajectory theory at near field, however support the trajectory theory at far field. Those novel phenomena provide the comprehensive information for further theoretical development and applications.
\end{abstract}

Keywords: double slit experiment, cross-double slit experiment, which-way-double-slit experiment, which-way-cross-double slit experiment, Bohr's complementarity principle, Bohm's trajectory theory

Content

1.Introduction

2. Experimental Test of Wave Description: Comprehensive Double Slit Experiments

2.1. Model, Two Rules and Two Postulates

2.2. Proof of Two Rules

2.3. Proof of Postulate-1

2.4. Proof of Postulate-2

3. Experimental Test of Trajectory Theory: Near Field and Far Field

3.1. Experiments Violating Trajectory Theory: Near Field:

3.2. Experiments Supporting Trajectory Theory: Far Field

3.3. Which-Way-Cross-Double-Slit Experiments Supporting Trajectory Theory: Far Field

4. Discussion and Conclusion

Appendix:

A1: Novel Diaphragm of Double Slit for Testing Trajectory Theory 


\section{A2. 2D-Cross-Double Slit Experiments}

A3. Which-Way-Cross-Double-Slit Experiments

\section{Introduction}

Young's double slit experiment was performed in 1801 [1,2], which, 100 years later, led to wave-particle duality. Feynman called it "a phenomenon [...] has in it the heart of quantum mechanics. In reality, it contains the only mystery [of quantum mechanics]" [3]. Moreover, the nature of photons truly puzzled Einstein. He wrote to M. Besso: “All these 50 years of conscious brooding have brought me no nearer to the answer to the question: What are light quanta?" [4].

To further explore the nature of photons, Young's double slit experiment was modified to which-way-double slit experiment by observing which slit a photon would pass through. Once which slit a photon passing through is determined, the wave-like interference pattern (hereafter, denoted as "pattern") disappears. The operational definition of "wave/particle" stands for "ability/inability to create interference" [5-7]. A variety of which-way-double slit experiments have been performed, such as double-double slit experiments [8].

To study the mystery of the double slit experiments and test both the wave description and trajectory description, cross-double slit experiments $[9,10]$ have been proposed/performed. In this article, we study the mystery by performing the comprehensive double slit experiments.

\section{Comprehensive Double Slit Experiment}

\subsection{Model, Two Rules and Two Postulates}

To analyze the double slit and cross-double slit experiments, we introduced the model and then, propose and demonstrate two rules and two postulates.

*) Model: the diaphragm of the double slit/cross-double slit and its right-side neighborhood is represented as a "virtual box" (Figure 2A). the width of the virtual box is to be determined experimentally.

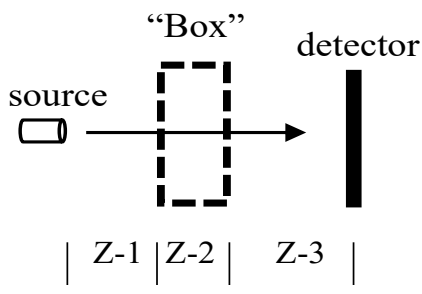

Figure 2A Double slit Apparatus with "virtual box"

Let us divide the model into 3 zones: zone-1 (Z-1) is from the source to the slide, which is the left 
boundary of the virtual box; the virtual box is zone-2 (Z-2); and zone-3 (Z-3) is from the right boundary of the virtual box to the detector.

*) Rule-1: When a single input beam of photons travelling as particles strikes a BS, the reflection and transmission attributed to the BS do not change the behavior of photons, namely, before and after the reflection and transmission, photons behave as particles.

*) Rule-2: When a light beam travelling as waves strikes a BS, reflection and transmission attributed to the BS do not change the behavior of photons, namely, before and after reflection by the BS and transmission through the BS, photons distribute as waves.

*) Postulate-1: in zone-1, photons behave as particles.

*) Postulate-2: in zone-3, photons behave as particles.

Postulate-1 predicts that before passing through the diaphragms of the double slit/cross-double slit, the light beam emitted by the laser source behaves as particles (photons), not as waves, which violates the standard wave theory describing the double slit experiments.

Postulate-2 predicts that before landing on the detector/screen, the light beam behaves as particles, not as waves. Namely, each fringe is formed independently and can be formed partially.

Indeed, the experimental results of testing the predictions of Postulate- 1 and -2 strongly support postulate-1 and -2 . Note that hereafter, to clearly show the results of experiments and draw conclusions without doubt, we arrange apparatuses to show the patterns created by photons passing through different paths on the same screen/detector. In Section 2, we study how photons behave in Z-1 and Z-3. We demonstrate Rule-1, Rule-2, Postulate-1 and Postulate-2 experimentally.

\subsection{Proof of Two Rules}

\subsubsection{Proof of Rule-1}

Experiment-2.1 (Figure 2.1)

Experimental setup: A laser source emits photons that are partially reflected by BS1 and arrive at detector 1 (D1) and partially transmitted though BS1 and arrive at D2. To show the multiple patterns on the same screen, we use a larger detector (Figure 2.1a).

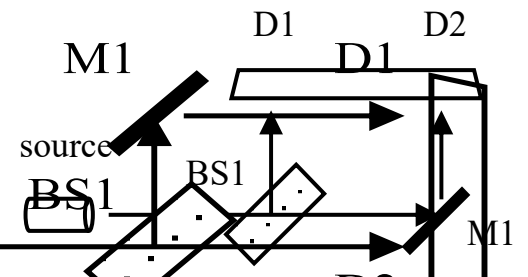

(a)

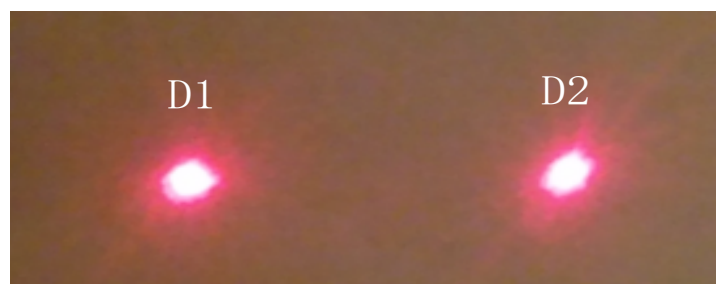

(b)

Figure 2.1. Testing Rule-1 
Observation (Figure 2.1b): both D1 and D2 show the images of the source.

Conclusion: Rule-1 is proved. Photons detected on both D1 and D2 have the same particle nature.

Namely the particle nature is not changed by either being reflected by BS or passing through BS.

\subsubsection{Proof of Rule-2}

Experiment-2.2 (Figure 2.2)

Experimental setup: A laser source emits photons that travel through a slide of cross-double slit. Then, the photons are partially reflected by BS1, partially transmitted through BS1, and arrive at D1 and D2 respectively (Figure 2.2a). The slide is a cross-double slit.

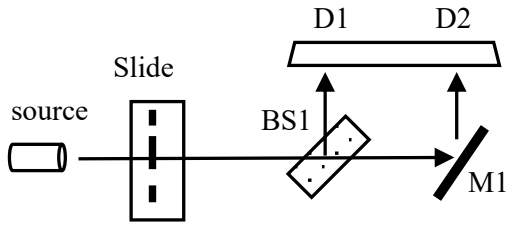

(a)

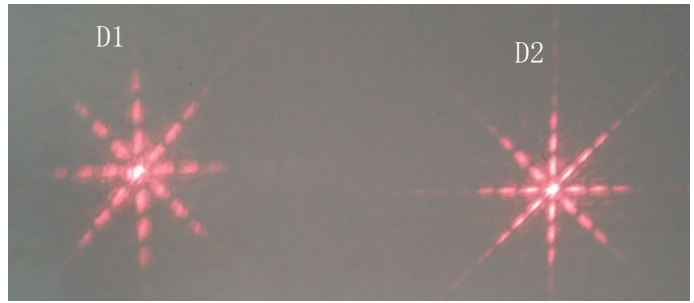

(b)

Figure 2.2 Testing Rule-2

Observation (Figure 2.2b): D1 and D2 show the same interference patterns.

Conclusions: Rule-2 is proved. Removing the slide, experiment-4.2 becomes experiment-4.1, which implies that it is the slide that changes photons' behavior. The combination of Rule-1 and Rule-2 shows that a BS does not affect the behavior of a single input beam of photons.

\subsection{Proof of Postulate-1}

The standard interpretation is that the light beam emitted by a source, e.g., a laser source, behaves as waves before passing through a double slit, i.e., in Z-1 (Figure 2B)
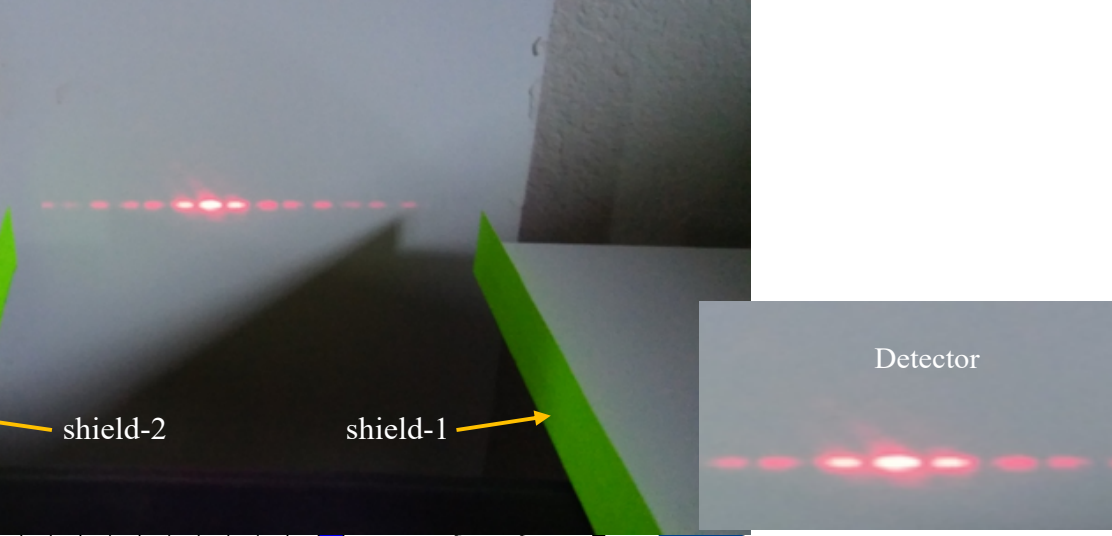

Figure 2B. Standard Interpretation of Double Slit Experiment

Postulate-1 says that the light beam behaves as particles in Z-1. Rule-1 and Rule-2 lead to Postulate-1. We perform three experiments to proof Postulate-1 (Experiment-2.3, -2.4 and -2.5). 
Experiment-2.3 (Figure 2.3): Demonstrate Postulate-1

Experimental setup (Figure 2.3a): Photons passing through BS1 and the slide arrive at D2. Photons reflected by BS1 arrive at D1.

D1

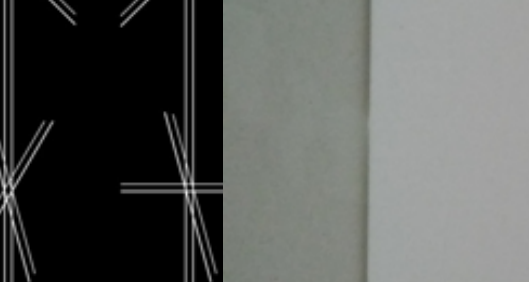

(a)

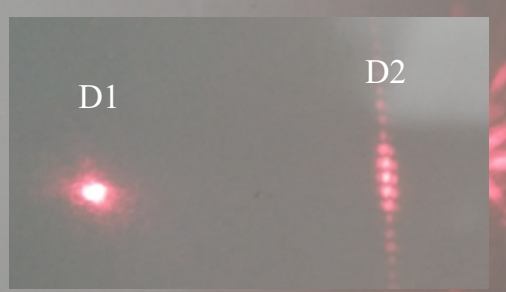

(b)

Figure 2.3. Demonstrate Postulate-1 (1)

Observations (Figure 2.3b): (1) D1 shows the image of the source; (2) D2 shows a pattern of the double slit.

Conclusion: D1 shows the particle nature of photons. Thus, based on Rule-1, photons passing through BS1 towards the slide of the double slit behave as particles as well, namely before arriving at the slide, photons behave as particles.

Postulate-1 is demonstrated.

Experiment-2.4 (Figure 2.4): Demonstrate Postulate-1

Experimental setup (Figure 2.4a): Photons travelling through BS1 and BS2, reflected by M2 and strike at D2. Photons reflected by BS1 and passing through slide-1 strike at D1. Photons reflected by BS2/M1/M3 and then passing through slide-3 strike at D3. To show the difference, we use a cross-double slit for slide- 1 and a double slit for slide-3.

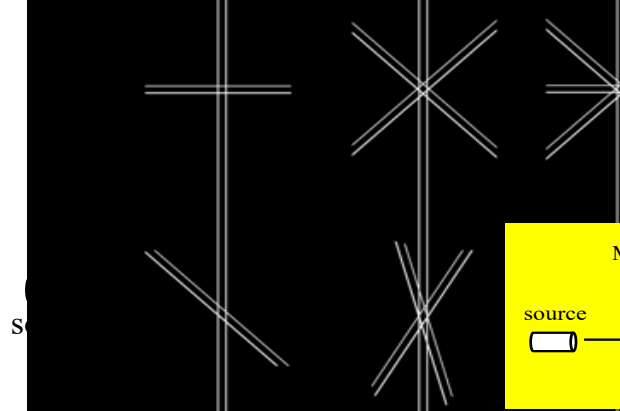

(a)

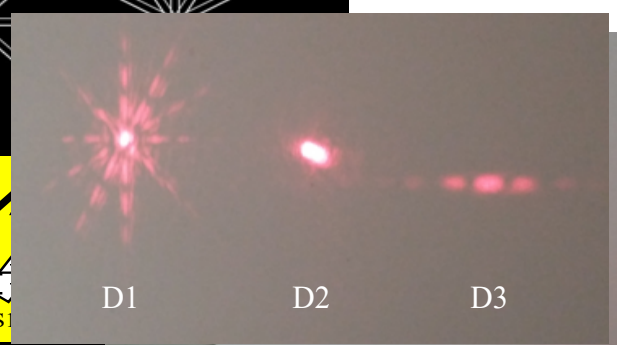

(b)

Figure 2.4. Testing Postulate-1 (2)

Observations (Figure 2.4b): D2 displays the image of the source, i.e., photons passing through BS1 and BS2 behave as particles. D1 and D3 show the wave distributions.

Conclusion: Photons passing through BS1/BS2 and reflected by M2 travel towards D2 and behave as particle. Thus, according to Rule-1, photons reflected by BS1 and by BS2/M1/M3 travel towards 
slide-1 and slide-3, respectively, behave as particles. Namely, photons behave as particles before passing through the double slit/cross-double slit.

Postulate-1 is proved again.

On the other hand, D1 and D3 show the wave distributions. Particle nature and wave distribution coexist in "the same experiment". To study wave-particle duality and complementarity principle, let us define the term, "the same experiment", as followings: when there is "only one source" emitting light/photons, the experiment is defined as the same experiment.

Experiment-2.5 (Figure 2.5): Demonstrate Postulate-1

Experimental setup (Figure 2.5a): Photons passing through BS1/BS2/BS3 and slide-4 arrive at D4. Photons reflected by BS1 arrive at D1; photons reflected by BS2 pass through slide-2 arrive at D2; photons reflected by BS3 arrive at D3.

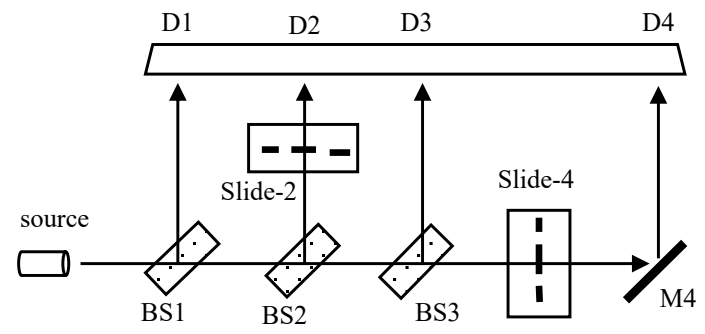

(a)

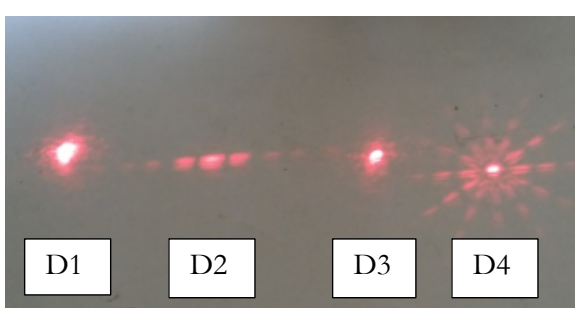

(b)

Figure 2.5 Double slit version of delayed choice experiment (1)

Observations (Figure 2.5b): D1 shows the image of the source; D2 displays the wave distribution; D3 shows the image of the source; D4 displays the wave distribution.

Conclusions: comparing Experiment-2.3, -2.4 and -2.5, we conclude that:

(1) Based on Rule-1, photons reflected by BS1 and BS3 behave as particles, thus, before passing through on slide-2 and slide-4, photons behave as particles.

(2) Slide-2 and slide-4 convert photons' particle behavior (before arriving) to wave distribution (after passing through) respectively.

(3) The slide determines the behavior of photons only when photons pass through it but not the behavior before passing it.

(4) The particle nature and wave distribution coexist in the same experiment.

\subsection{Proof of Postulate-2}

\subsubsection{Plan of Proving Postulate-2}

First, let us adopt the standard interpretation that the light beam behaves and interferes as waves before landing on the screen/detector, i.e., in Z-3, as shown by wave-shaped-curves in Z-3 of Figure $2 \mathrm{C}$. 
Then we use several methods, e.g., inserting shields and blockers in Z-3, to interrupt the waves and to see how the patterns change. If the patterns did change, then the light beam behaves as waves in Z-3. Otherwise, the light beam does not behave as waves in Z-3.

An analogy is the breakwater that breaks the coming water waves.

Figure $2 \mathrm{C}-\mathrm{b}$ show that placing shield-1 contacting/near the detector. One can move shield-1 towards to the slide of the double slit/cross-double slit to determine the right-boundary of Z-2 (Figure 2C-a). To insert blockers in Z-3 to check how the fringes show (Figure 2C-c). We can also place the combinations of shields and blockers in Z-3 as shown in Figure 2C-d, 2C-e and 2C-f.

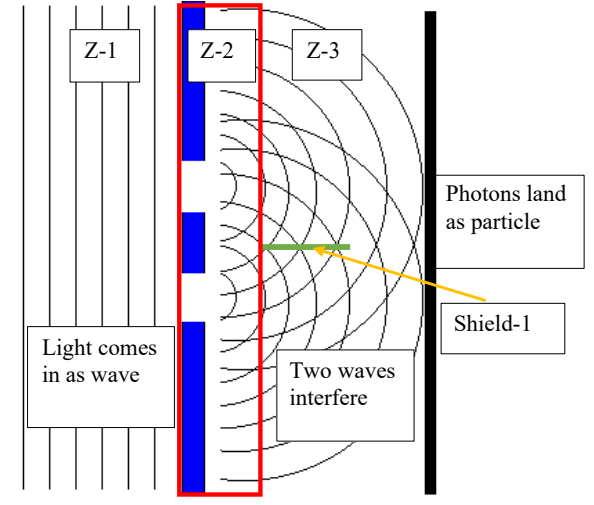

(a)

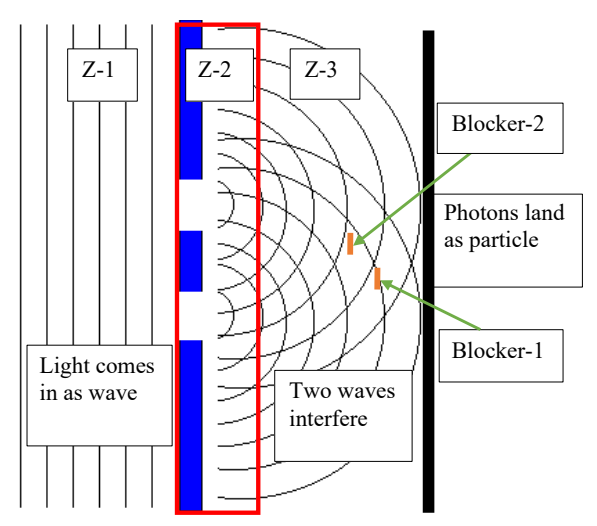

(c)

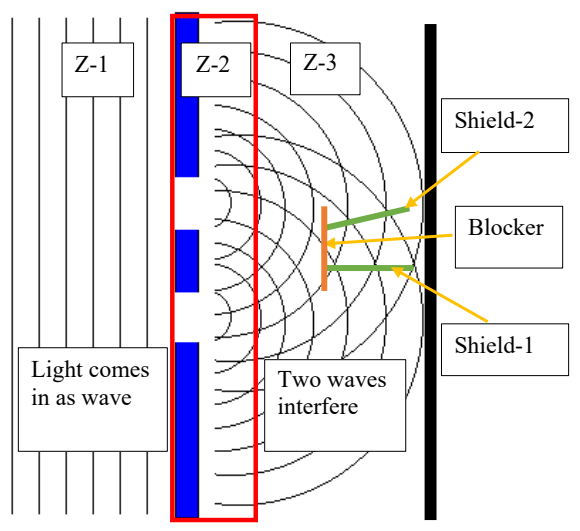

(e)

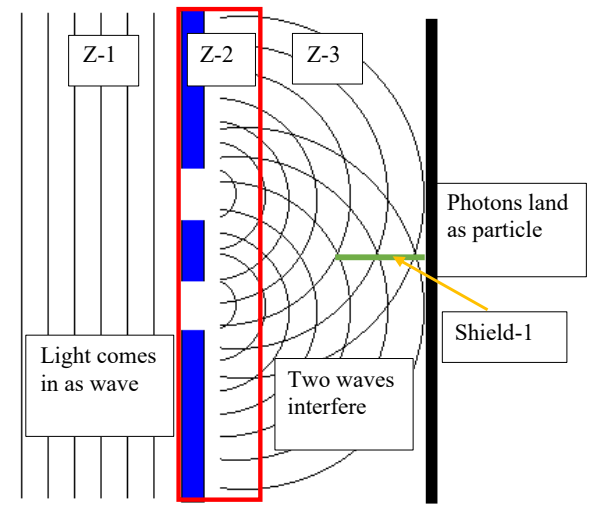

(b)

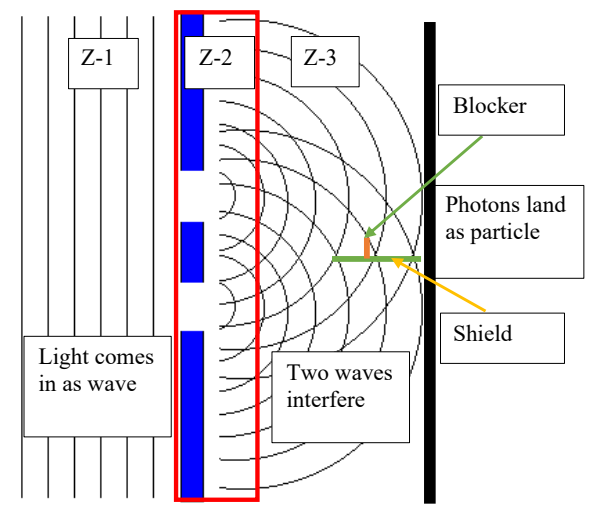

(d)

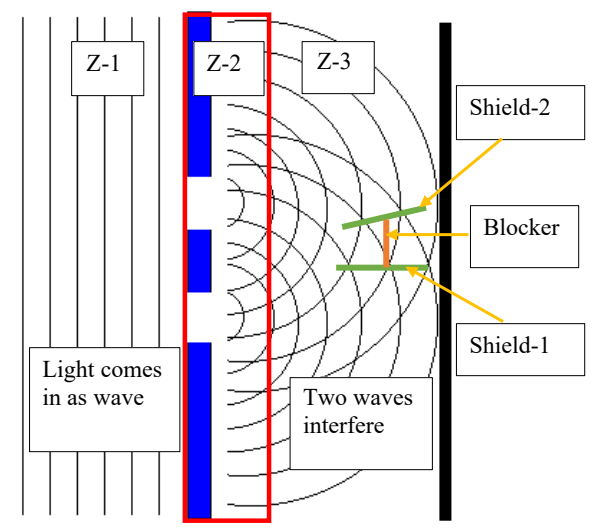

(f)

Figure 2C. Schematics for proofing Postulate-2 


\subsubsection{Proof of Postulate-2: with Shield Near Detector}

Experiment-2.6 (Figure 2.6): testing postulate-2 with a Shield.

The experiment is performed in two setups.

Experimental setup-1 (Figure 2.6-1a): Shield-1 contacts the detector.

Placing a "shield" (green colored) made of carboard in Z-3 of the regular double slit experiment contacting the detector. The purpose is to test whether the shield would separate waves and thus prevent photons from interfering if photons would behave as waves in Z-3.

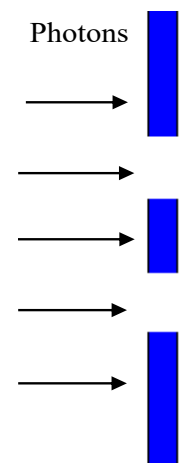

(a)

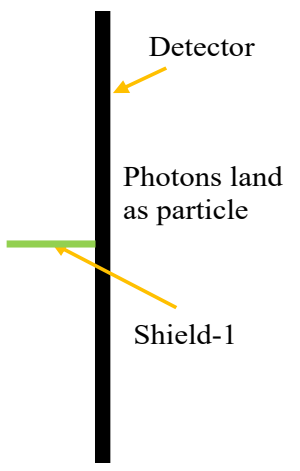

Figure 2.6-1 Testing Postulate-2 with Single Shield (1)

For simplicity, shield-1's orientation is from the center of the double slit points to the center of the zeroth-order fringe. We refer shield-1 as longitudinal. Shield-1 is 28 inches long, 1.5 inch wide, and 0.3 mm thick. The distance between the double slit and the detector is 200 inches. An analogy is a breakwater that break water waves.

Observation (Figure 2.6-1b): The interference pattern keeps no noticeable change, namely, shield-1 does not affect the interference pattern, which would not be expected if photons behave as waves.

Experimental setup-2 (Figure 2.6-2a): Shield-1 is one inch away from the detector.

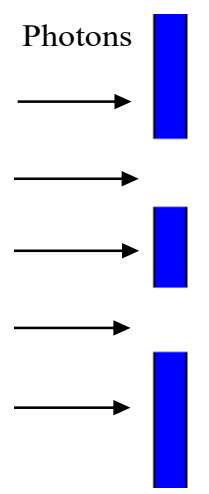

.(a)

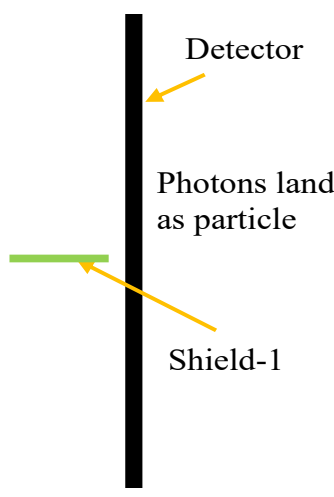

Figure 2.6-2 Testing Postulate-2 with Single Shield (2)

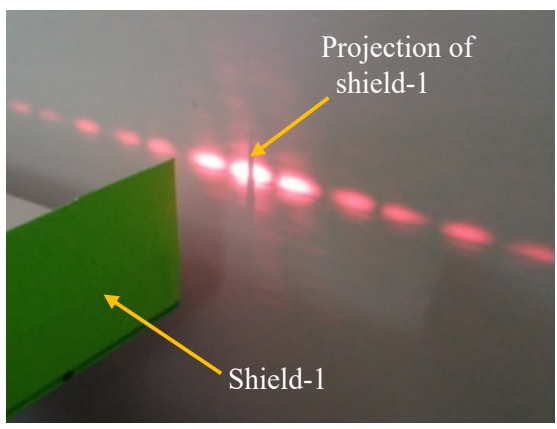

(b) 
Observation (Figure 2.6-2b): (1) Shield-1 does not affect the interference pattern; (2) there is the projection of shield-1 at the middle of the zeroth-order fringe.

Conclusion: The phenomena of experimt-4.6 would be expected only if photons behave as particles. Postulate-2 is demonstrated.

Experiment-2.7 (Figure 2.7): Testing Postulate-2 with two Shields (1)

Introducing shield-2. Two shields form a narrow channel. The purpose is to test whether the channel would prevent photons from interfering if photons would behave as waves in Z-3. Shield-2 is 28 inches long, 1.5 inch wide, and $0.3 \mathrm{~mm}$ thick.

The experiment is carried out in three setups.

Experimental setup-1 (Figure 2.7-1a): Both shield-1 and shield-2 contact the detector.

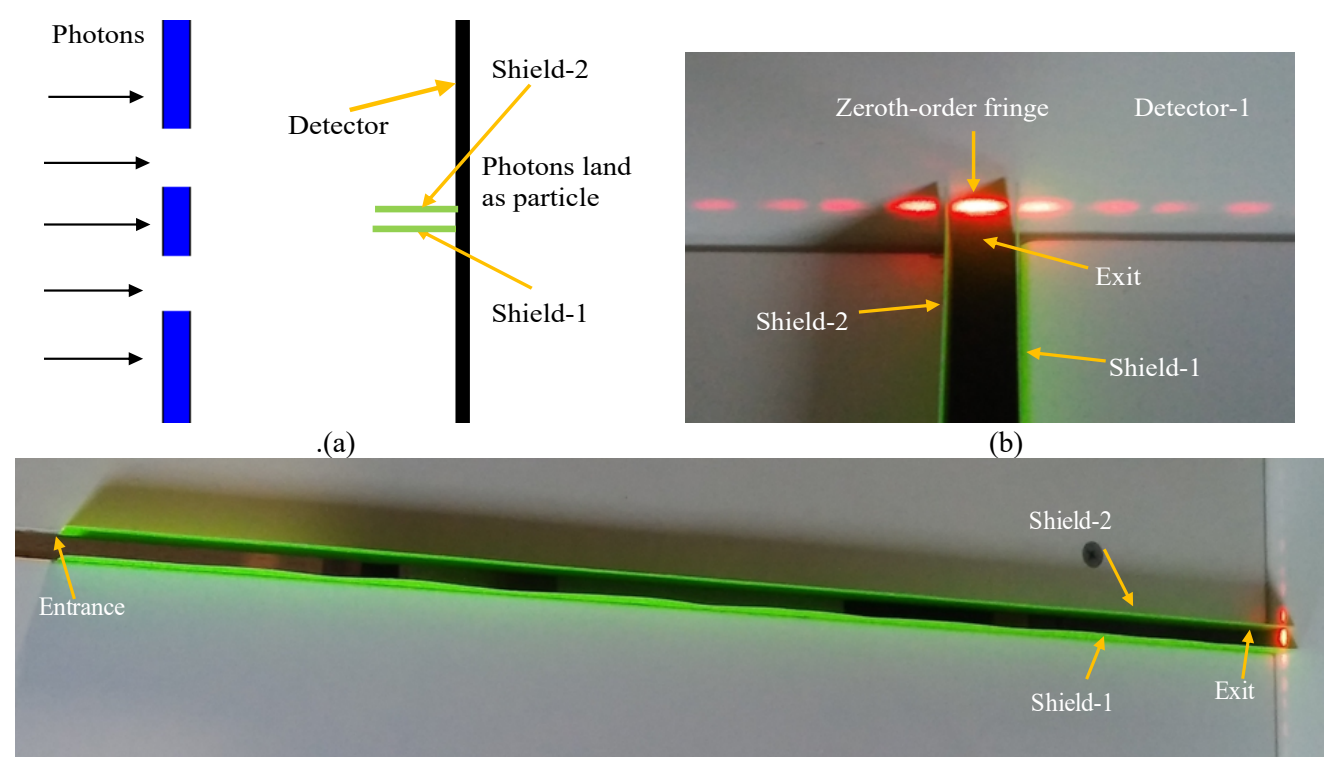

(c)

Figure 2.7-1 Testing Postulate-2 with Two Shields

Observation (Figure 2.7-1b): We observe the interference pattern that is the same as that there were no shield-1 and shield-2. The existence of two approximately parallel shields of 28 inches long has no effect on the "interference" pattern of $650 \mathrm{~nm}$ light, which indicates that photons do not behave as waves near the detector.

Experimental setup-2 (Figure 2.7-2a): Moving shield-1 and Shield-2 one inch away from the detector. 


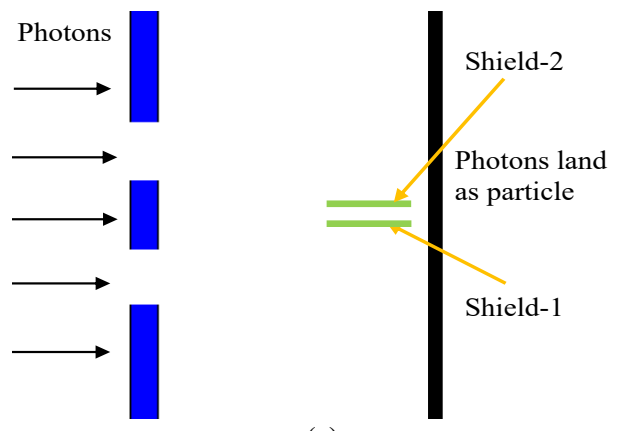

(a)

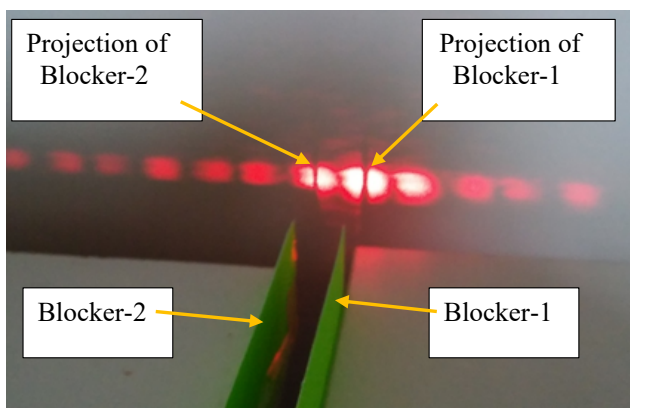

(b)

Figure 2.7-2 Testing Postulate-2 with Two Shields

Observation (Figure 2.7-2b): the interference pattern has no change. The projection of shield-1 appears at the middle of the zeroth-order fringe, while the projection of shield-2 appears at the middle of the first-order fringe. Only photons behaving as particles can (1) pass through the narrow channel; (2) strike at the positions of the zeroth-order fringe and a first-order fringe on the detector; (3) form two projections, while (4) do not disturb the existing interference pattern.

Experimental setup-3 (Figure 2.7-3a): Picture of two shields 70 inches long contacting to the detector. Note that the picture was shot from the "Entrance" to the detector so that the pattern and apparatus show on the same picture and thus, Entrance looks wider.

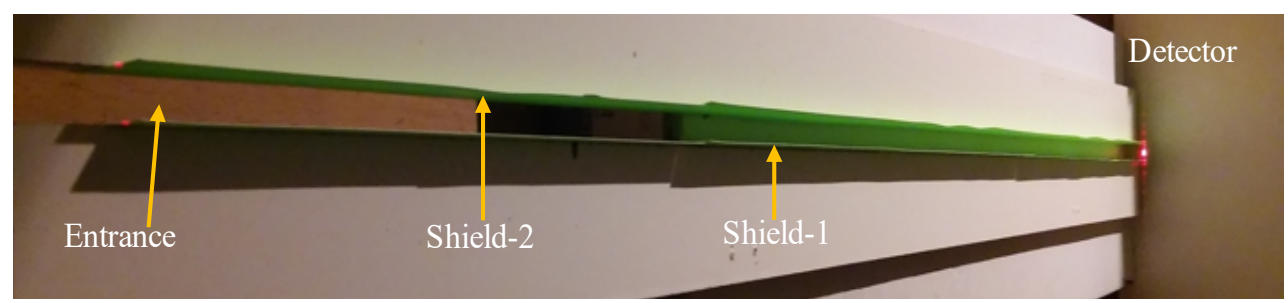

(a)

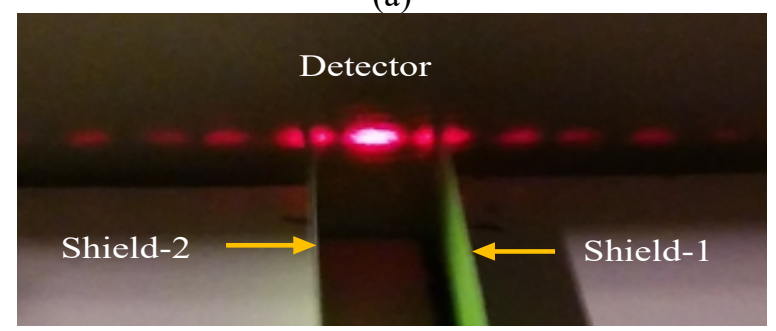

(b)

Figure 2.7-3 Testing Postulate-2 with Two Shields of 70 inches long

Observation (Figure 2.7-3b): the interference pattern is the same as if there were no shield-1 and shield-2. The existence of two long shields has no effect on the interference pattern. Two projections are show at the $m=+1$ and $m=-1$ fringes.

Observations indicate that photons behave as particles.

Experiment-2.8 (Figure 2.8): Testing Postulate-2 with two Shields (2)

Experimental setup (Figure 2.8a): Move shield-2 to 60 inches from double slit; shield-1 stays. 


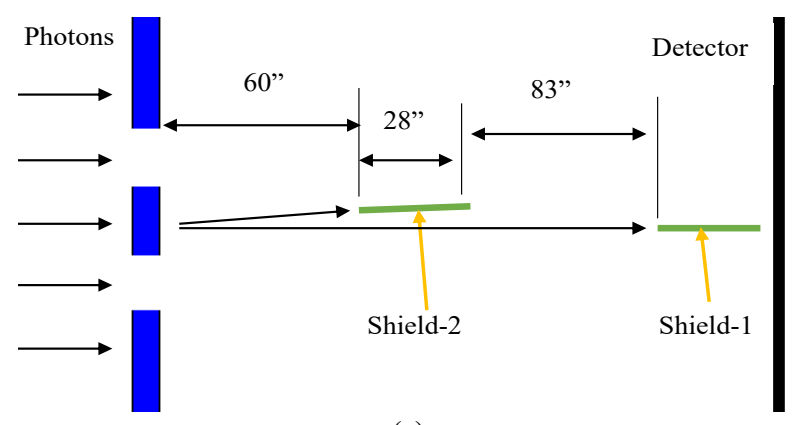

(a)

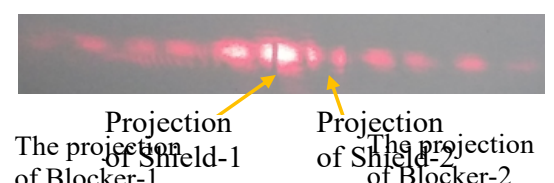

(b)

Figure 2.8 Testing Postulate-2 with Two Shields (2)

Observation (Figure 2.8b): The interference pattern has no change. The projection of shield-2 is wider than that of shield-1 since it is closer to the double slit.

Conclusion: Postulate-2 is experimentally confirmed. Only particles can pass through the long and narrow channel between shield- 1 and shield- 2 and form fringes on the detector; thus, photons behave as particles long before landing on the detector. On the other hand, photons are distributed with a wave-like interference pattern on the detector. The phenomena of "wave-particle coexistence" would lead to a deeper understanding of the double slit experiments. Comprehensive double slit experiments have been carried out at different positions, for example, 40 inches, 80 inches, and 120 inches away from the detector. We always observe the pattern and projections of shield-1 and shield-2.

\subsubsection{Proof of Postulate-2: with Blockers Near Detector}

Experiment-2.9 (Figure 2.9): Testing Postulate-2 with Blocker.

Let us consider five experimental setups.

Experimental Setup-1 (Figure 2.9-1a): blocker-10, blocker-11 and blocker-12, each is 0.5-inches wide, are placed along the normal vector of the detector, and separated by 4 inches.

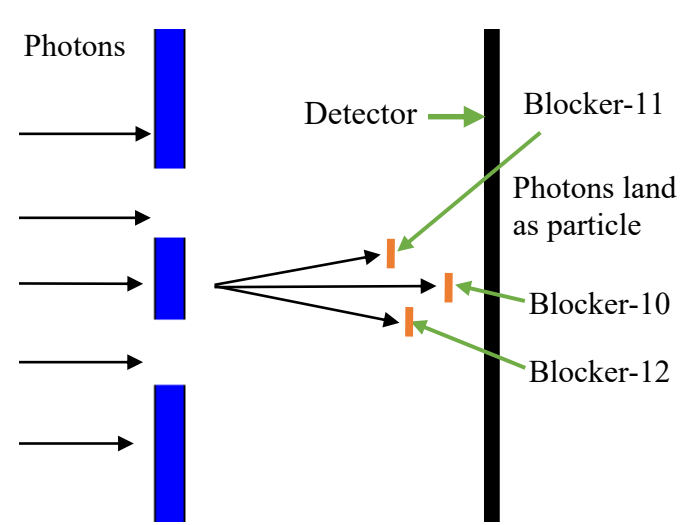

(a)

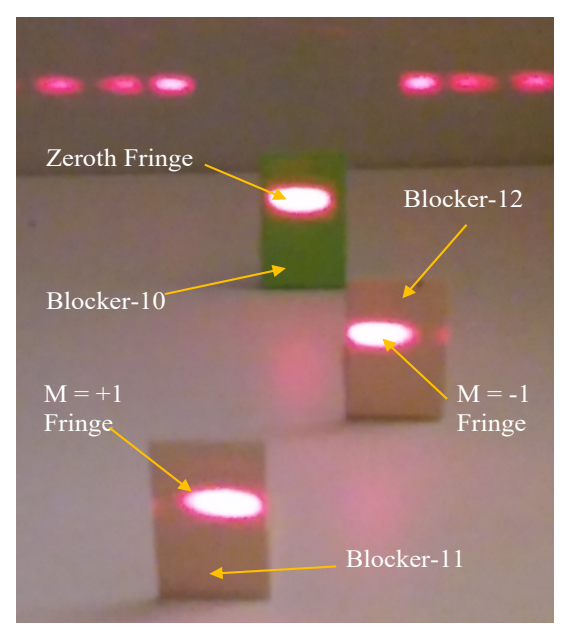

(b)

Figure 2.9-1 Fringes Formed Independently (1) 
Observation (Figure 2.9-1b): Three blockers are arranged such that the zeroth-order fringe and two first-order fringes are formed on blocker-10, blocker-11 and blocker-12, respectively. The existence of each blocker does not affect the fringes formed on other blockers and on the detector. Namely, fringes are formed independently.

Experimental Setup-2 (Figure 2.9-2a): blocker-11 and blocker-12 are placed along the normal vector of the surface of the detector and separated by 4 inches.

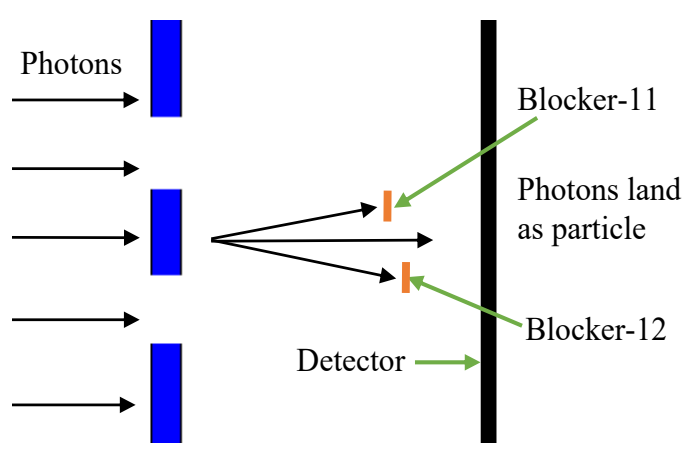

(a)

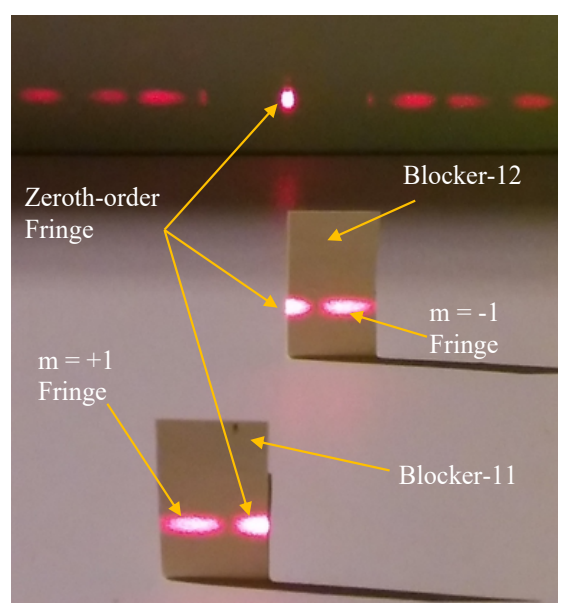

(b)

Figure 2.9-2 Fringes Formed Independently and Partially (2)

Observation (Figure 2.9-2b): Two blockers are arranged such that portions of the zeroth-order fringe are formed on the detector, blocker-11 and blocker-12. Thus, the fringe can be formed partially. The existence of each blocker does not affect the fringes formed on other blockers and on the detector. Namely, fringes are formed independently.

Experimental Setup-3 (Figure 2.9-3): blocker-11 and blocker-12 are placed along the normal vector of the surface of the detector.

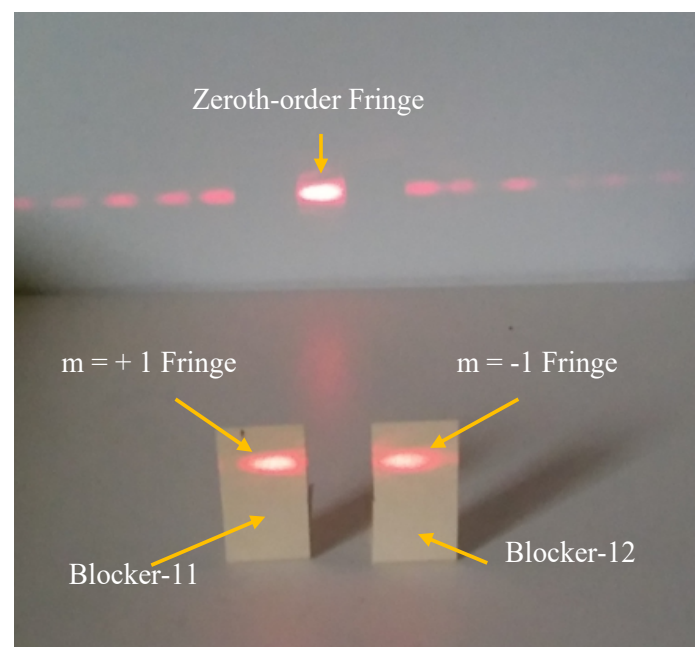

Figure 2.9-3 Fringes Formed Independently (3) 
Observation: Zeroth-order fringe, $\mathrm{m}=+1$ fringe and $\mathrm{m}=-1$ fringe are formed on the detector, blocker-11 and blocker-12, respectively.

Experimental Setup-4 (Figure 2.9-4): blocker-11 and blocker-12 are placed along the normal vector of the surface of the detector, and separated by 4 inches.

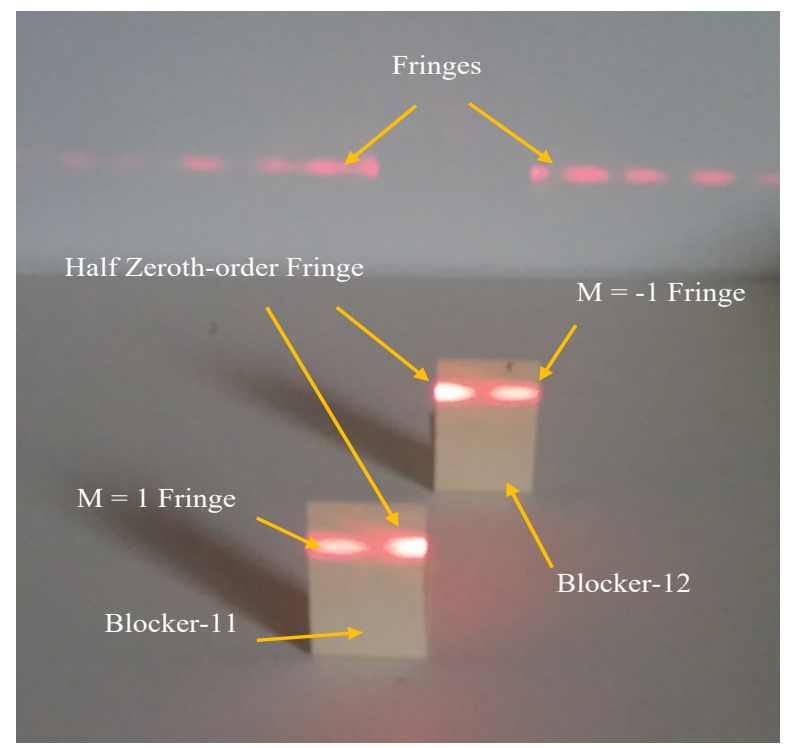

Figure 2.9-4 Fringes Formed Independently and partially (4)

Observation (Figure 2.9-4): Portions of the zeroth-order-fringe are formed on blocker-11 and blocker-12, which indicates that the fringe can be partially formed. The $\mathrm{m}=+1$ fringe and $\mathrm{m}=-1$ fringe are formed on blocker-11 and blocker-12, respectively, i.e., formed independently.

Experimental Setup-5 (Figure 2.9-5): blocker-11 and blocker-12 are placed along the normal vector of the surface of the detector and separated by 4 inches.

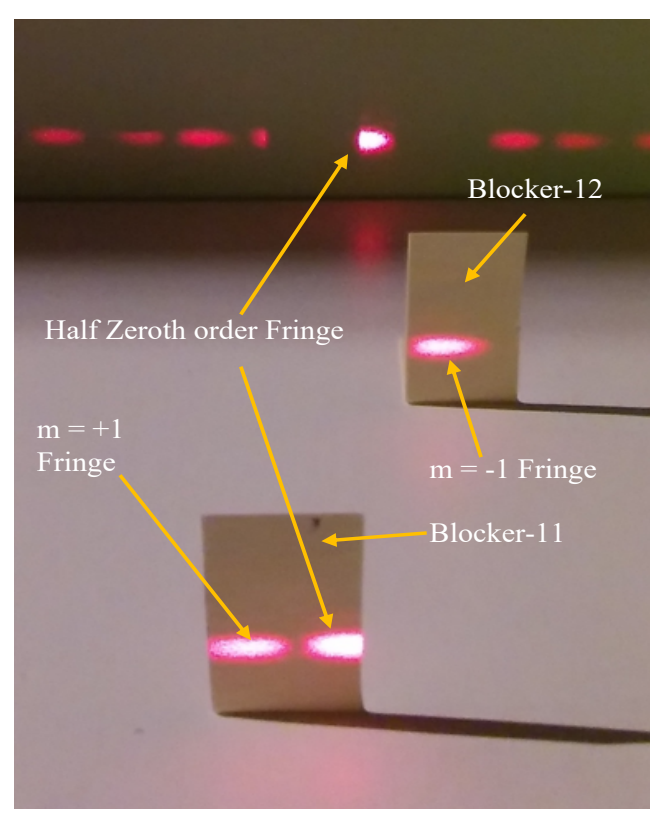

Figure 2.9-5 Fringes Formed Independently and partially (5) 
Observation (Figure 2.9-5): Portions of the zeroth-order fringe are formed on the detector and blocker-11, which indicates that the fringe can be partially formed. The $\mathrm{m}=+1$ fringe and $\mathrm{m}=-1$ fringe are formed on blocker-11 and blocker-12, respectively, i.e., formed independently.

Conclusion: Fringes are formed independently and partially, which would be expected only if photons behave as particles in Z-3 near the detector.

Postulate-2 is experimentally confirmed.

Some of photons form fringes on blockers, while some of photons are distributed like partial of a wave interference pattern on the detector.

\subsubsection{Proof of Postulate-2: Shields and Blocker.}

We have shown that, on the one hand, longitudinal shield(s) do not disturb the wave distribution in zone-3. On the other hand, blockers do block the propagation of photons as photons are particles. Now let's show the effects of combinations of shields and blockers.

Experiment-2.10 (Figure 2.10-1, 2.10-2, 2.10-3): Testing Postulation-2 with Shield and Blocker. The experiment is performed in three setups.

Experimental setup-1 (Figure 2.10-1-a): Shield is one inch away from detector. Blocker is positioned next to shield.

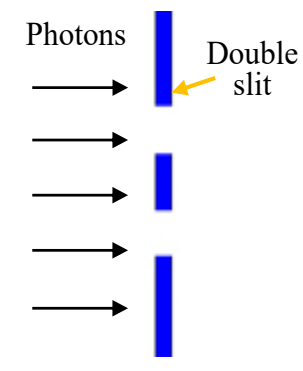

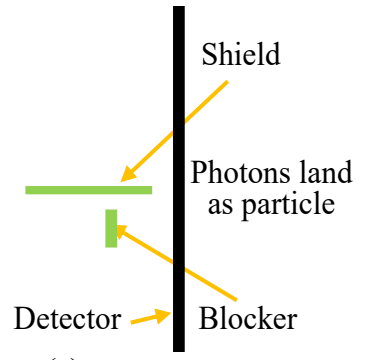

(a)

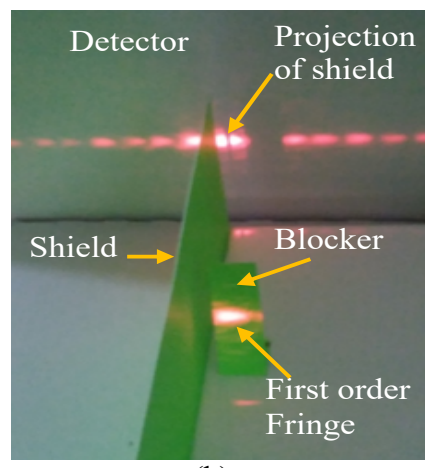

(b)

Figure 2.10-1 Testing Postulate-2 with Shield and Blocker (1)

Observation (Figure 2.10-1-b): (1) Shield does not affect the interference pattern at all; (2) there is the projection of the shield at the middle of the zeroth-order fringe; (3) blocker is so arranged that it does block a first-order fringe.

Experimental setup-2 (Figure 2.10-2-a): Shield is one inch away from detector. Blocker-1 and blocker-2 are placed on both sides of shield respectively. 


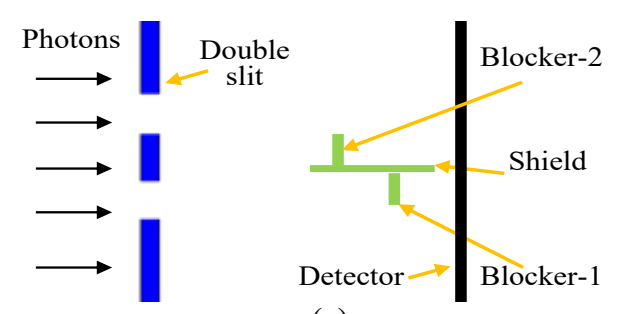

(a)

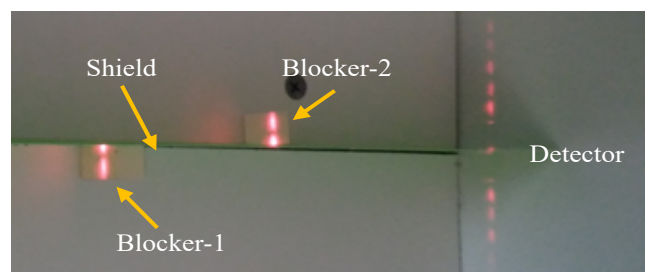

(b)

Figure 2.10-2 Testing Postulate-2 with Shield and Blocker (2)

Experimental setup-3 (Figure 2.10-3-a)

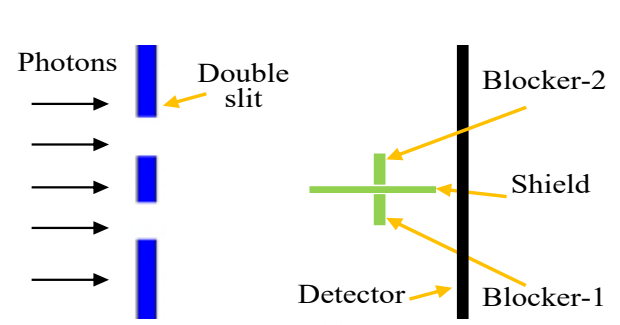

(a)

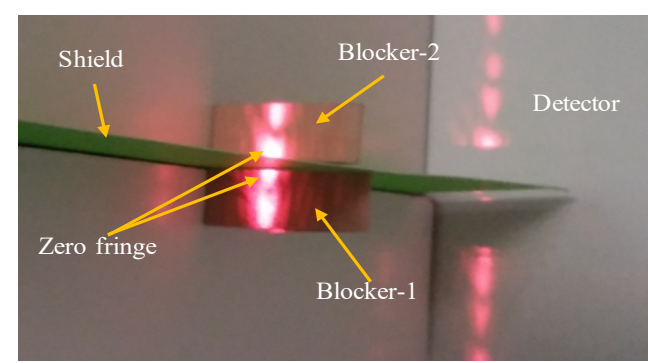

(b)

Figure 2.10-3 Testing Postulate-2 with Shield and Blockers (3)

Observation (Figure 2.10-2-b and 2.9-3-b): Blocker-1 and Blocker-2 are so arranged that the zeroth-order fringe is formed on both blocker-1 and blocker-2 respectively. Two first-order fringes are formed on blocker-1 and blocker-2, next to the half of the zeroth-order fringe, respectively. Shield can divide the zeroth-order fringe into two parts, but cannot disturb the remaining fringes, which indeed indicates that photons behave as particle before landing on detector

Experiment-2.11 (Figure 2.11-1, 2.11-2, 2.11-3): Testing Postulation-2 with Shields and blocker.

We perform this experiment in three setups.

Experimental Setup-1 (Figure 2.11-1a): place blocker-1 at the other end of both shield-1 and shield-2, where we denote it as Entrance (Figure 2.11-1b).

Observation (Figure 2.11-1b): The interference pattern is formed on blocker-1 instead of the detector.
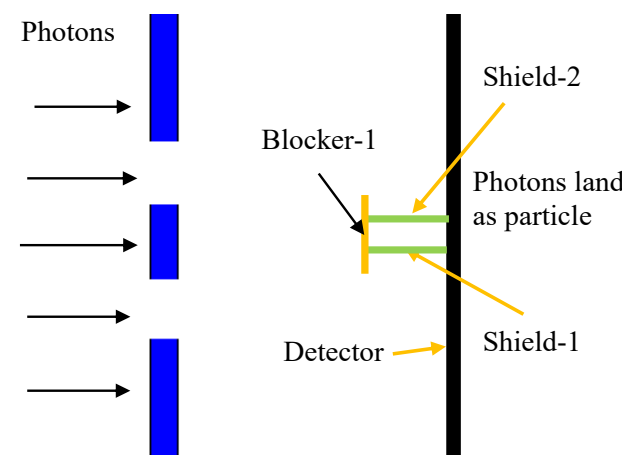

(a)

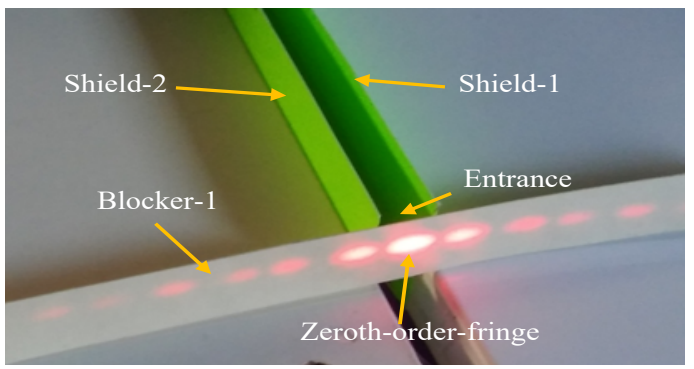

(b)

Figure 2.11-1 Testing Postulate-2 with Shields and Blocker

Experimental Setup-2 (Figure 2.11-2a): Cutting the top portion of blocker-1. 


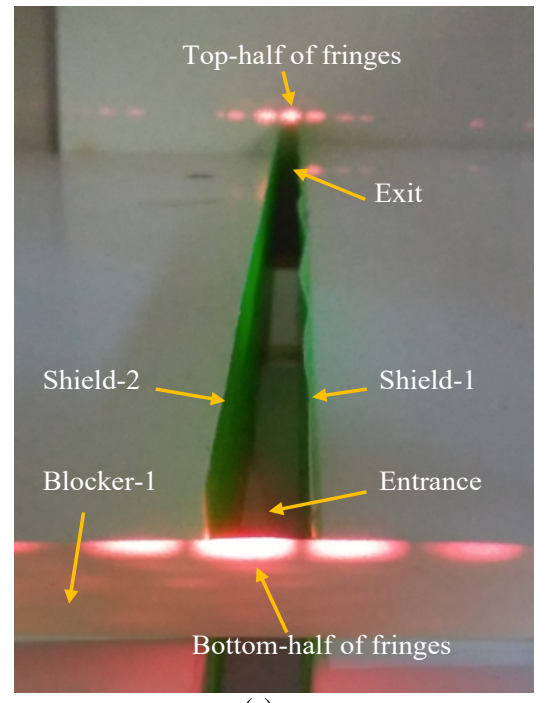

(a)

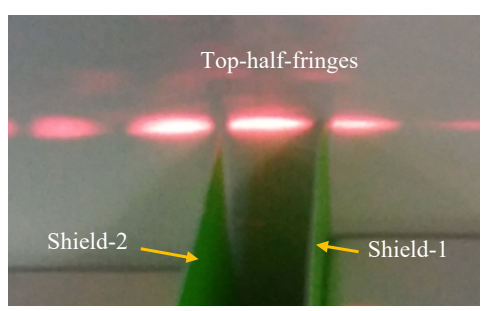

(b)

Figure 2.11-2 Testing Postulate-2: Cut Top Half of Blocker-1

Observation (Figure 2.11-2): the bottom half of the fringes still show on blocker-1, while the top half show on the detector. Namely each fringe can be formed partially. And shields have no effect on the positions of the interference pattern at all.

Experimental Setup-3 (Figure 2.11-3): cut a "U" shape gap at the position of the zeroth-order fringe on blocker-3.

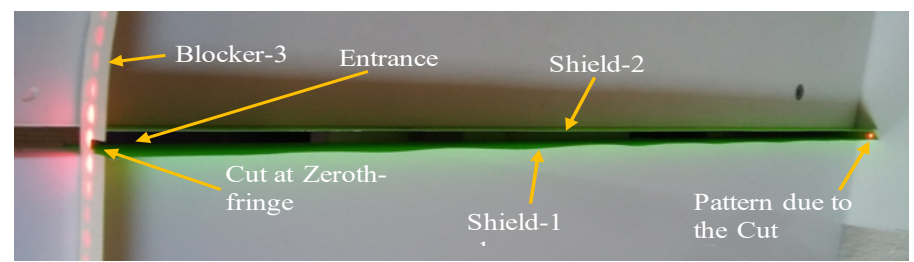

(a)

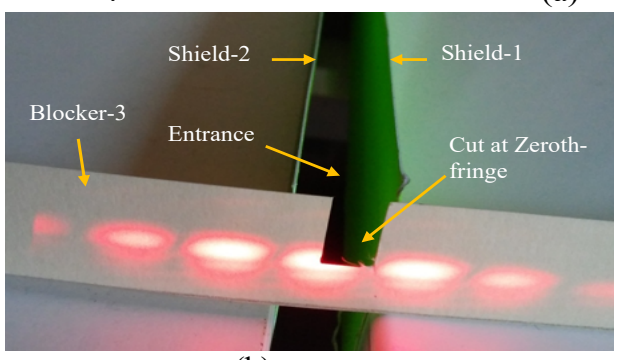

(b)

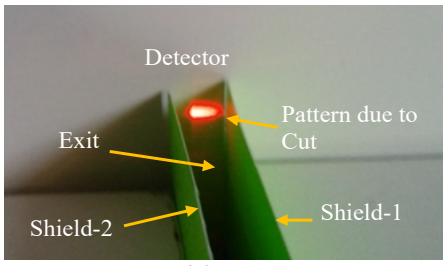

(c)

Figure 2.11-3 Testing Postulate-2: Blocker-3 with Cut

Observation (Figure 2.11-3b and 2.11-3c): Photons pass through the cut and form the exactly same shape of patterns on the detector, which shows the particle nature of light and that photons move along straight lines.

Conclusion: each fringe can be formed partially and individually, which can be expected only if photons are particles

Experiment-4.12: Testing Postulate-2 with Shields and Blocker. 
We perform this experiment in two setups.

Experiment Setup-1 (Figure 2.12-1a): insert transverse blocker-2 one inch wide into the channel formed by shield-1 and shield-2.
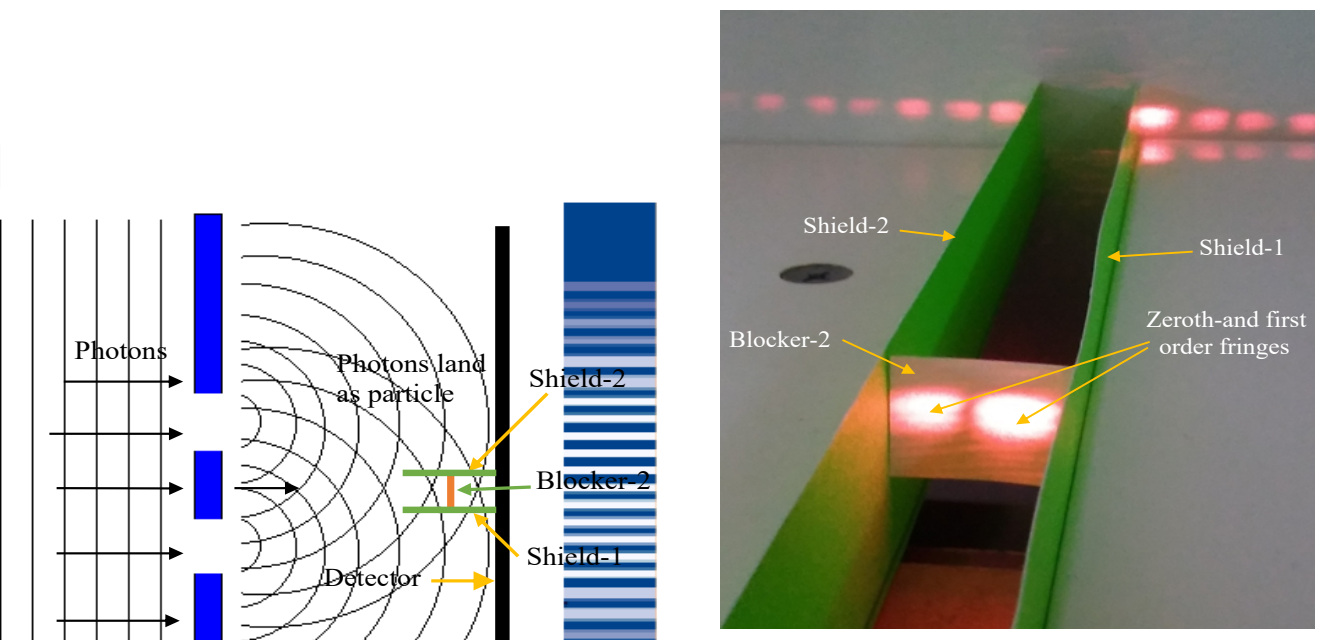

(a)

(b)

Figure 2.12-1 Blocker-2 in Channel (1)

Observation (Figure 2.12-1b): Two fringes are formed on blocker-2, and the remaining fringes are formed on the detector. Namely, Fringes can be formed independently. Two shields have no effect on the interference pattern. This observation indicates that photons behave as particles.

Experimental Setup-2 (Figure 2.12-2): cut two triangles on blocker-2 at the locations of the zeroth-order fringe and a first-order fringe respectively. Then place blocker-2 into the channel.

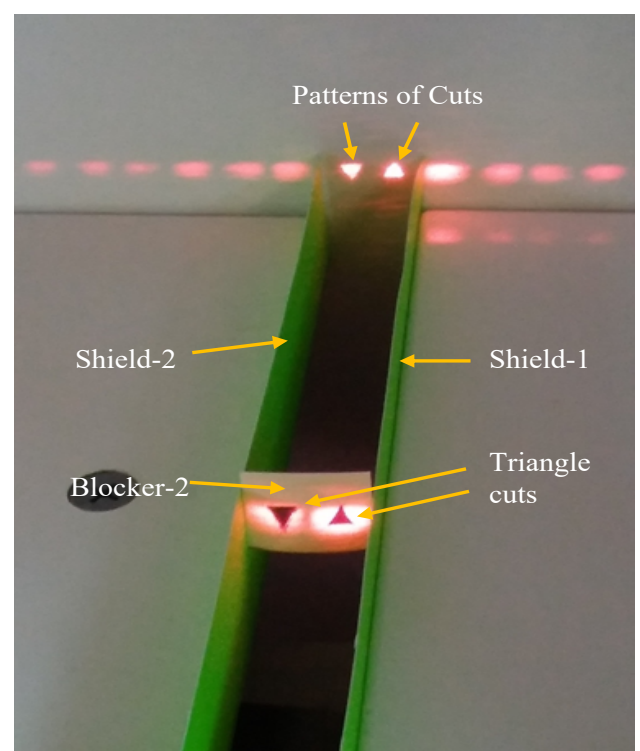

Figure 2.12-2 Blocker-2 with Two Cuts (2)

Observation (Figure 2.12-2): Photons pass through two triangle-shaped cuts and form exactly the same triangle-shaped patterns on the detector, which shows the particle nature of photons, namely 
photons move along straight lines. Note that photons are not directly from the source; they are just pass through a double slit and were supposed to behave as waves.

Conclusion: The conclusion is that photons behave as particles.

Postulate- 2 is confirmed experimentally.

Meanwhile, some of photons are distributed like a partial wave pattern on the detector. Namely the particle nature and wave distribution coexist.

\subsubsection{Proof of Postulate-2: 2D-cross-double slit}

Without losing generality, we perform comprehensive double slit experiments with two cross-double slit that consist of five and six double slits crossing at the same spot. Each of double slit creates an interreference pattern independently.

Experiment-2.13 (figure 2.13-1 to 2.13-4): The experiment is performed in four setups.

Experimental Setup-1 (Figure 2.13-1): parallel shield-1 and shield-2 contact the detector.

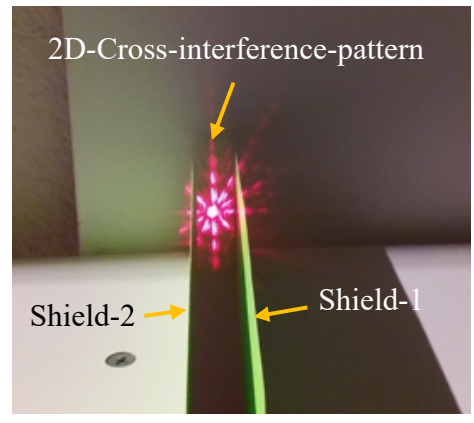

Figure 2.13-1. 2D-pattern vs. Channel (1)

Observation: the channel of two shields does not disturb the 2D-interference pattern.

Experimental setup-2 (Figure 2.13-2a): using blocker-3 to block the bottom portion of the

2D-interference pattern.

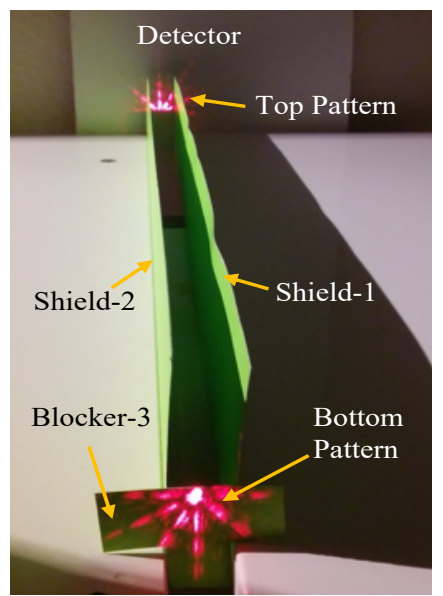

(a)

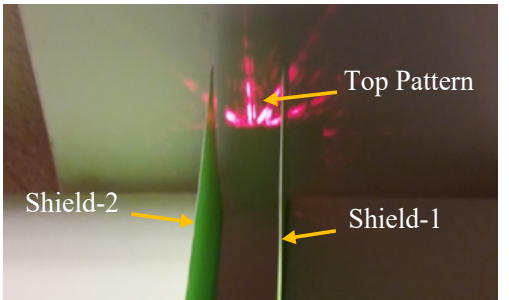

(b)

Figure 2.13-2. Testing Postulate-2 with Cross-double slit (2) 
Observation (Figure 2.13-2b): the top portion of the 2D interference pattern is shown on the detector, while the bottom portion is shows on blocker-3.

Experimental setup-3 (Figure 2.13-3): using blocker-3 to block bottom-right corner of the 2D-interference pattern.

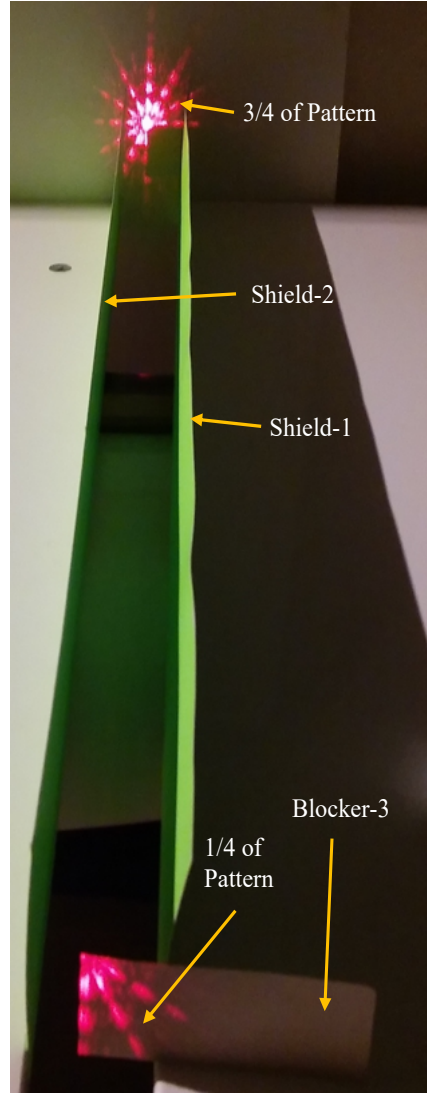

Figure 2.13-3

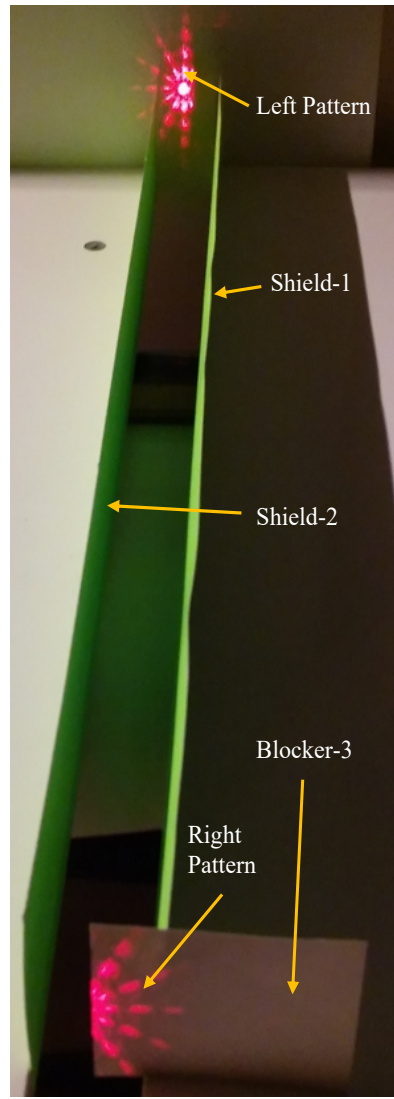

Figure 2.13-4

Observation (Figure 2.13-3): the $3 / 4$ portion of the 2D interference pattern shown on the detector, while the bottom-right portion of the interference pattern shows on blocker-3.

Experimental setup-4 (Figure 2.13-4): using blocker-3 to block right portion of the 2D-interference pattern.

Observation (Figure 2.13-4): the left portion of the 2D interference pattern shows on the detector, while the right portion of the interference pattern shows on blocker-3.

Conclusion: 2D patterns are created independently and partially. Only particle can behave in such way.

Postulate-2 is confirmed experimentally: in zone-3, photons move along predetermined trajectories to form fringes and thus, behave as particles.

It is a challenge to consistently interpret the experiments in Section 4.

\section{Experimental Test of Trajectory Theory: Near Field and Far Field}


de Broglie and Bohm theory [11-13] states that photons propagate along trajectories. One prediction of computer simulations of de Broglie-Bohm theory is that the possible trajectories for particle (Figure 3A-a, extracted from reference [14-16] and for photons (Figure 3A-b, extracted from reference [14]) cannot cross.

We notice an implicit prediction that there is a triangle-shaped area behind the double slit, in which there is no trajectory (Figure 3A-a and 3A-b), namely, no particle/photons pass through this area (hereafter referred both as "predictions").

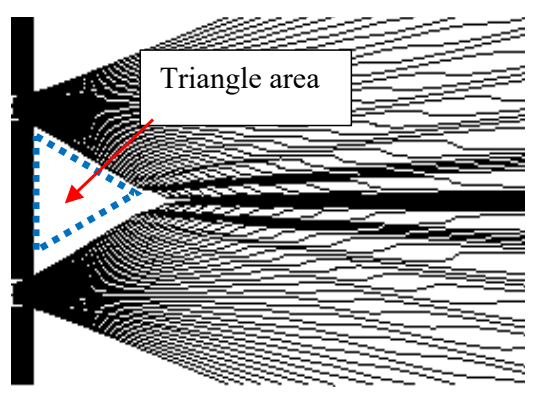

(a)

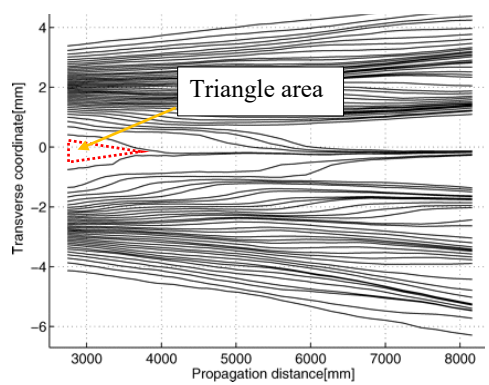

(b)

Figure 3A Computer simulations of trajectory interpretation of double slit experiments

To our knowledge, no experiment has either been performed or proposed to test these two predictions.

In this Section, we perform more comprehensive double slit experiments to test two predictions of computer simulations of Bohm trajectory theory in Z-2.

\subsection{Experiments Violating Trajectory Theory: Near Field:}

Shield Contacting Diaphragm of Double Slit

We observed that the height of the triangle is longer than the spacing between two slits. Thus, we test whether there are photons in the triangular area using shield (Figure 3B).

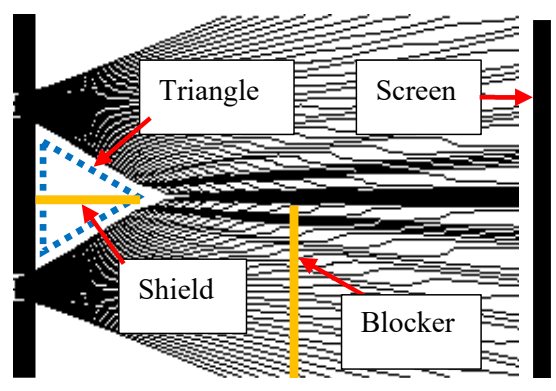

Figure 3B. Outline of experiments

Note that in Figure 3B, we use Figure 3A-a for a schematic drawing of photon experiments instead of Figure 3A-b for photons. The conclusions are the same. We perform two experiments to test whether there are photons in the triangular area.

Experiment-3.1 (Figure 3.1-1 and 3.1-2): we show that, with a narrow shield, the trajectories cross, while the wave distribution exists. 
Experimental Setup (Figure 3.1-1): making a shield (gray colored) and gluing it to an object (Figure 3.1-1b). The shield is $2.8 \mathrm{~mm}$ long, $9.5 \mathrm{~mm}$ wide, and $0.12 \mathrm{~mm}$ thick. Note that the drawing is not to scale.

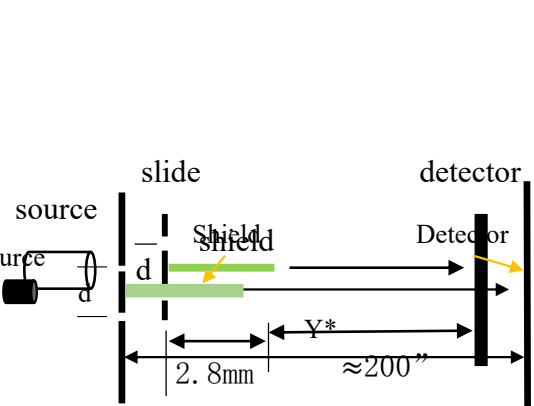

(a)

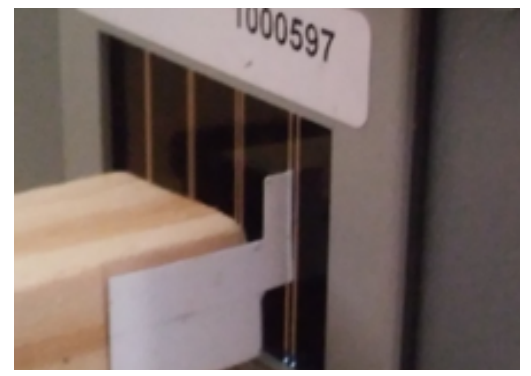

(c)

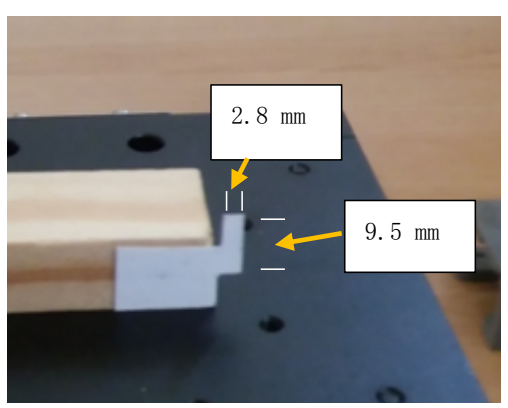

(b)

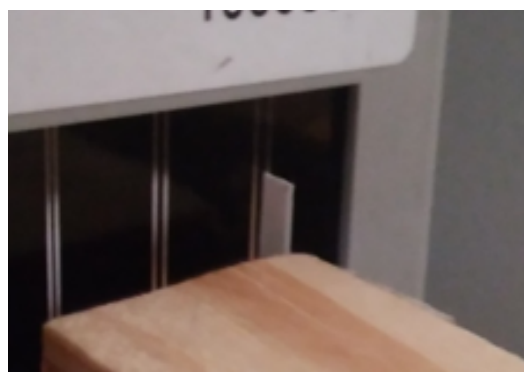

(d)

Figure 3.1-1. Experimental setup

Place the shield along the virtual centerline and contacting the double slit at the position between two slits, where the spacing " $\mathrm{d}$ " between two slits is $1 \mathrm{~mm}$ (Figure 3.1-1a). Figure 3.1-1c is the picture taken from the right side of the shield, and Figure 3.1-1d is the picture taken from the left side.

Note that the "contact" is macroscopic "contact", namely there are actually "gaps" between the shield and the double slit. More precise apparatus is designed in Appendix.

Then turning on the laser.

Observation: the light from the right-side slit shins on the right side of the shield (Figure 3.1-2a), while the light from the left-side slit shins on the left side of the shield (Figure 3.1-2b). The light spots on both sides of the shield indicate that there are light/photons in the triangle area.

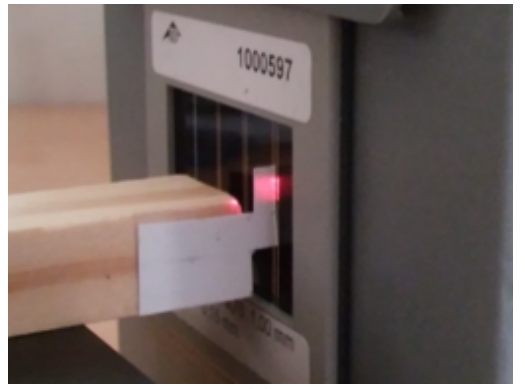

(a)

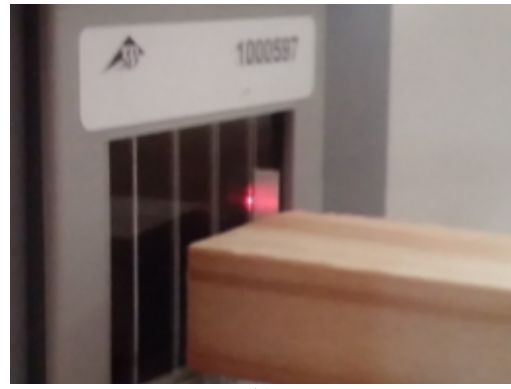

(b)

Figure 3.1-2 Experiments with Shield Contacting Double Slit 


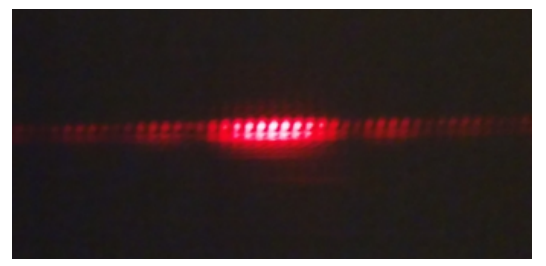

(a)

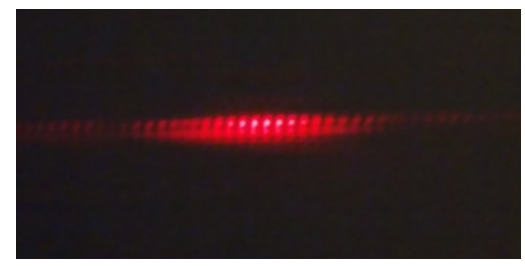

(b)

Figure 3.1-3 Regular interference pattern vs. interference pattern of double slit with shield

Observation: Figure 3.1-3a shows the interference pattern of the double slit without the shield. Figure $3.1-3 b$ is the interference pattern of the same double slit with the shield.

Experiment-3.2 (Figure 3.2-1 and 3.2-2): with a wider shield, the trajectories cross and the interference pattern exists.

Experimental Setup (Figure 3.2-1): making an "L"-shaped shield (grey colored) and gluing it to an object (Figure 3.2-1b). The shield is $25 \mathrm{~mm}$ (one inch) long, $8 \mathrm{~mm}$ wide, and $0.12 \mathrm{~mm}$ thick.

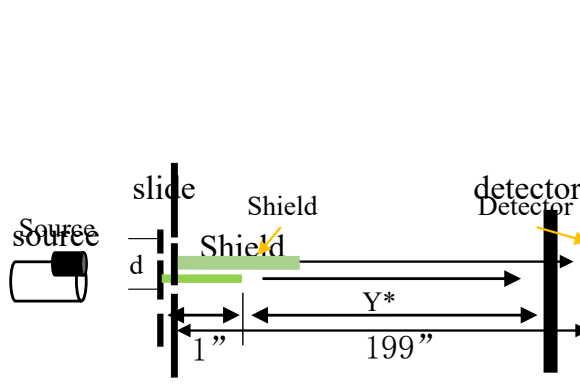

(a)

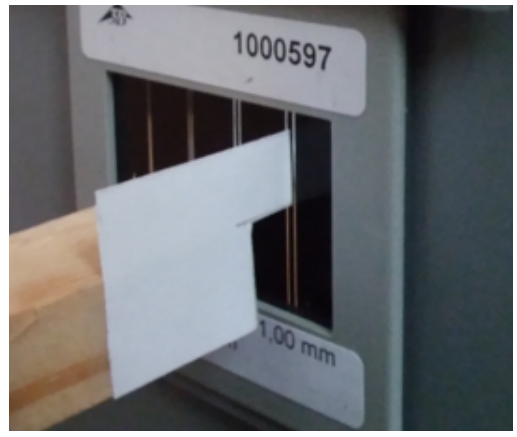

(c)

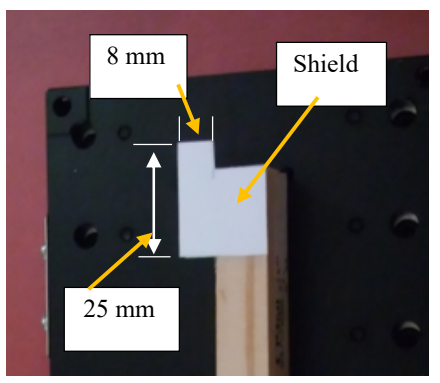

(b)

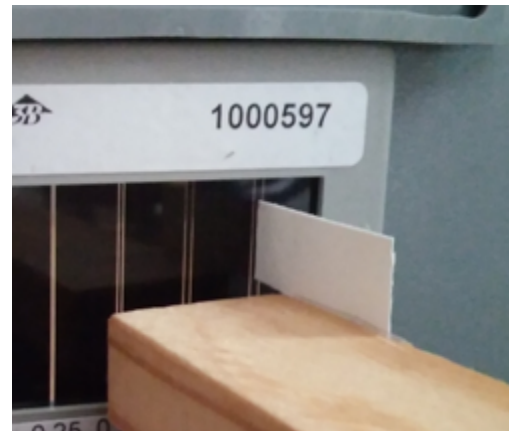

(d)

Figure 3.2-1 Schematic and Shield

The shield is placed along the virtual centerline and contacts the double slit at the point between two slits (Figure 3.2-1c and 3.2-1d), where the spacing "d" between two slits is $1 \mathrm{~mm}$ (Figure 3.2-1-a).

Then turning on the laser. 


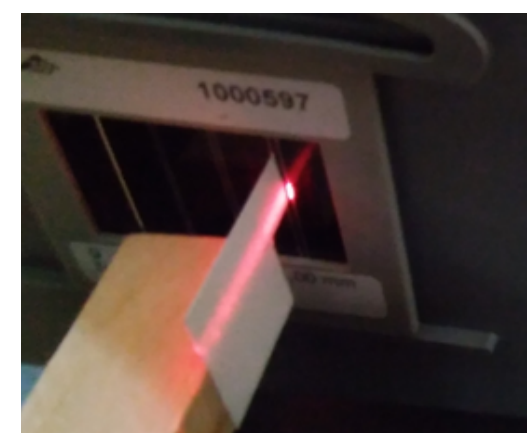

(a)

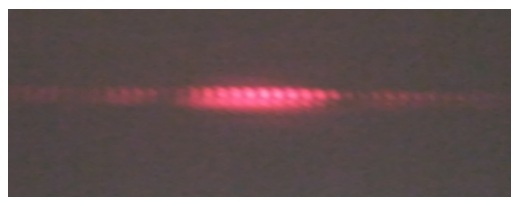

(c)

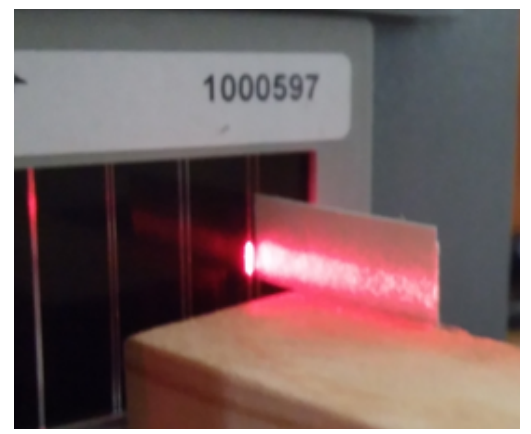

(b)

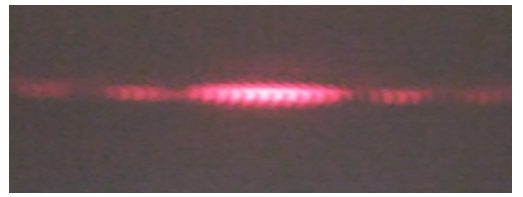

(d)

Figure 3.2-2. Experiment with shield contacting double slit

Observation: the light from the right-side slit shins on the right side of the shield (Figure 3.2-2a),

while the light from the left side slit shines on the left side of the shield (Figure 3.2-2b). The light spots on both sides of the shields indicate that there are light/photons in the triangle area. The shield is brightest close to the slits. The interference pattern exists (Figure 3.2-2c), but is dimmer. For comparison, Figure 3.2-2d shows the interference pattern of the same double slit, but without the shield.

Conclusion: in the triangular area, first, there are light/photons; second, trajectories cross. Thus, we suggest that the experimental observations challenge either the predictions of computer simulation or the trajectory theory.

It is reasonable to assume that, without the shield (Figure 3C-b), the behavior of light/photons in and near the triangular area would be the same as that with the shield (Figure 3C-a).

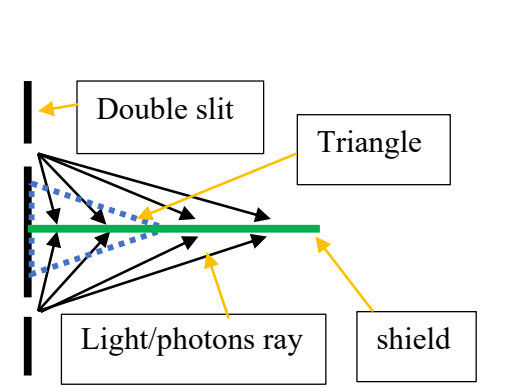

(a)

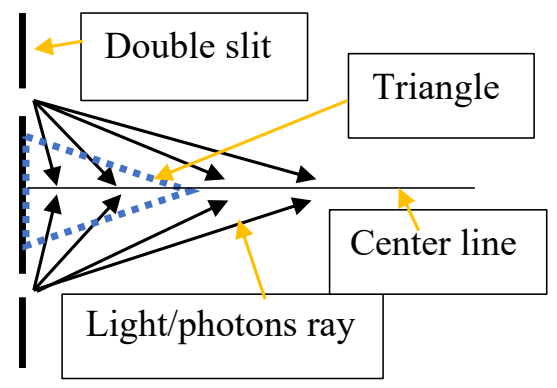

(b)

Figure 3C. Schematics of Experiment

\subsection{Cross-Double Slit Experiments Supporting Trajectory Theory: Far Field}


The purpose of the experiments below is to test the behaviors of light/photons in Z-3 near the detector. In the following experiments, the blocker was 128 inches away from the diaphragm of the double slit. To avoid losing generality, we perform experiments with a cross-double slit. First, let us show the interference pattern of a cross-double slit experiment without a blocker.
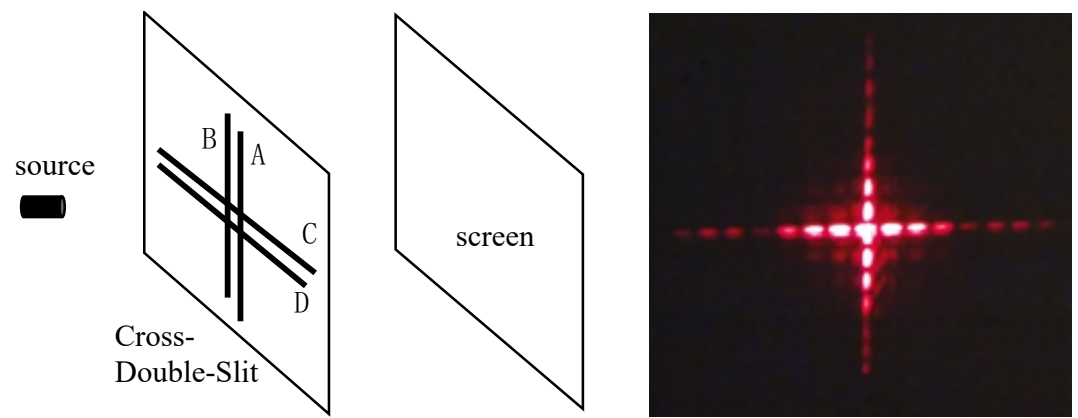

Figure 3D Cross-double slit experiment

Experiment-3.3 (Figure 3.3): Place blocker-AB and/or blocker-CD to block the different portions of the interference pattern created by double slit $\mathrm{AB}$ and double slit-CD. The experiment is performed in three setups.

Experimental setup-1 (Figure 3.3-1a): Placing blocker-AB such that it blocks the left half of the interference pattern, except the zeroth-order fringe.

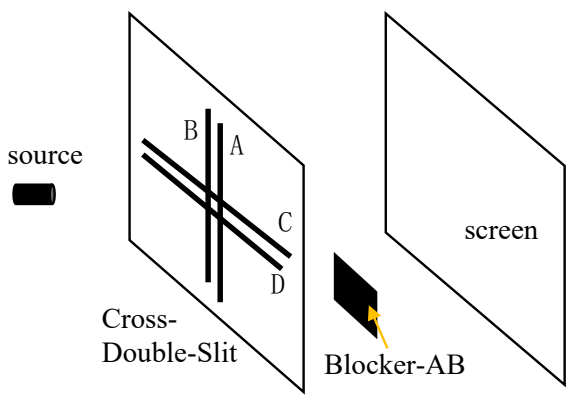

(a)

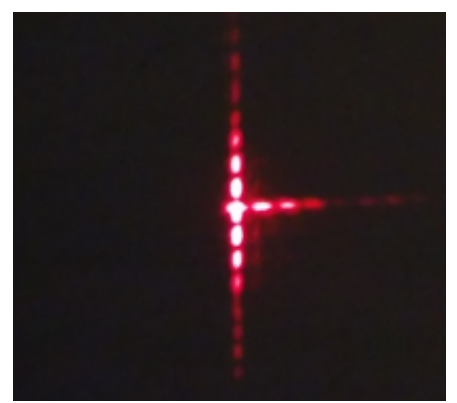

(b)

Figure 3.3-1 Block left half of the interference pattern of double slit-AB

Observation (Figure 3.3-1b): the left half of the fringes are blocked. The positions of the right-half fringes of the interference pattern are not affected by the blocker. The positions of the fringes in the vertical interference pattern are not affected. This is consistent with the statement that the photons move along trajectories.

Experimental setup-2 (Figure 3.3-2a): Placing blocker-CD such that it blocks the bottom half of the interference pattern created by double slit-CD. 




(a)

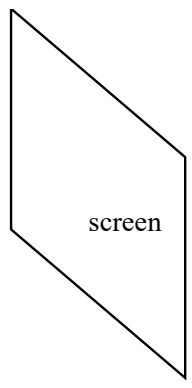

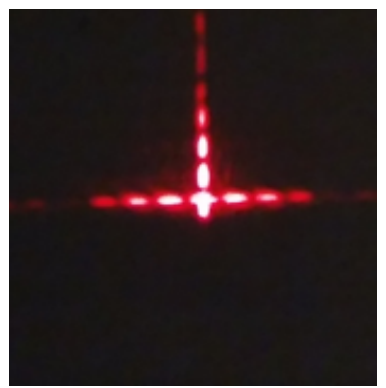

(b)

Figure 3.3-2 Block bottom half of the interference pattern of double slit-CD

Observation (Figure 3.3-2b): the bottom half of the fringes are blocked. The remaining fringes are not affected by blocking, which shows that photons move along trajectories.

Experimental setup-3 (Figure 3.3-3a): Placing both blocker-AB and blocker-CD to block the different portions of the $1 \mathrm{D}$ interference patterns created by double slit- $\mathrm{AB}$ and double slit-CD, respectively.

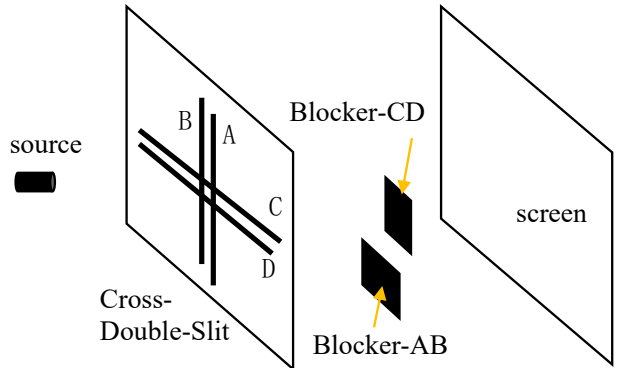

(a)

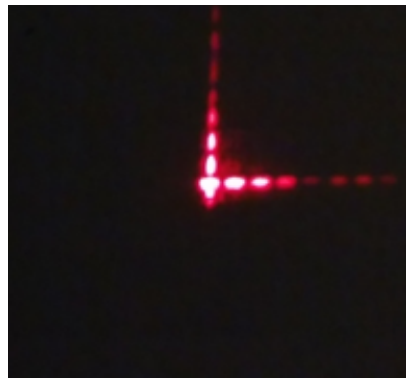

(b)

Figure 3.3-3 Blocking half of interference patterns created by both double slit-AB and double slit-CD Observation (Figure 3.3-3b): (A) the bottom half fringes created by double slit-CD are blocked; and (B) the left-half fringes created by double slit-AB are blocked. The remaining fringes are not affected by the existence of the blockers, which shows that photons move along trajectories.

The tilt-cross-double slit apparatus is employed, which consists of a vertical double slit-AB and a tilt-double slit-CD crossing to each other. Figure 3E shows the pattern without blocker.

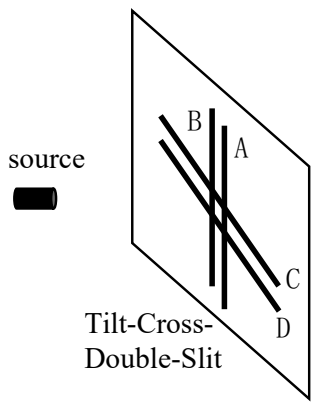

(a)

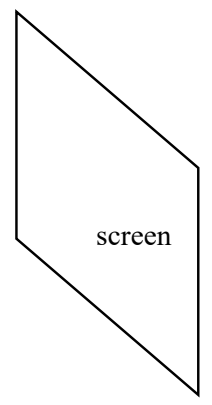

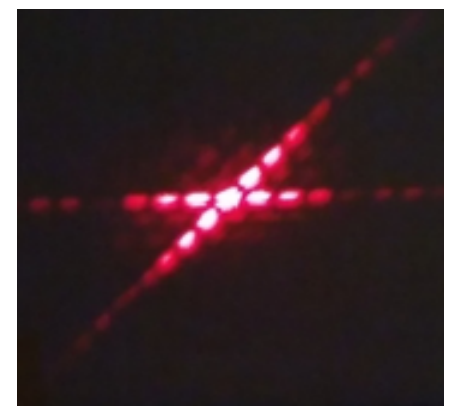

(b)

Figure 3E Tilt cross-double slit experiment 
Experiment-3.4 (Figure 3.4): Tilt-cross-double slit experiments. Blocker-AB is placed to block the different portions of the interference pattern created by double slit AB. The experiment is performed in three setups.

Experimental setup-1 (Figure 3.4-1a): Placing blocker-AB such that it blocks half of the interference pattern created by double slit-AB.

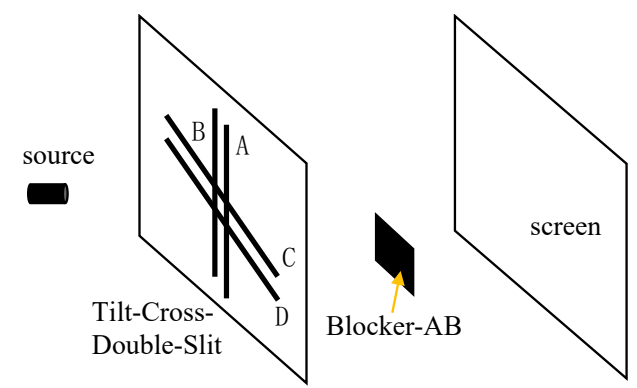

(a)

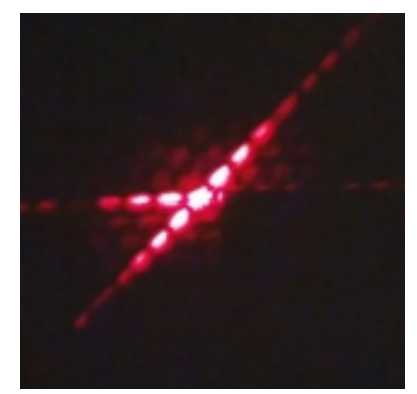

(b)

Figure 3.4-1 Tilt cross-double slit experiment with blocker-AB

Observation (Figure 3.4-1b): the horizontal right half fringes are blocked. The remaining fringes are not affected by blocking. The observations show photons move along trajectories.

Experimental setup-2 (Figure 3.4-2a): Placing blocker-CD such that it blocks the bottom half of the interference pattern created by double slit-CD.

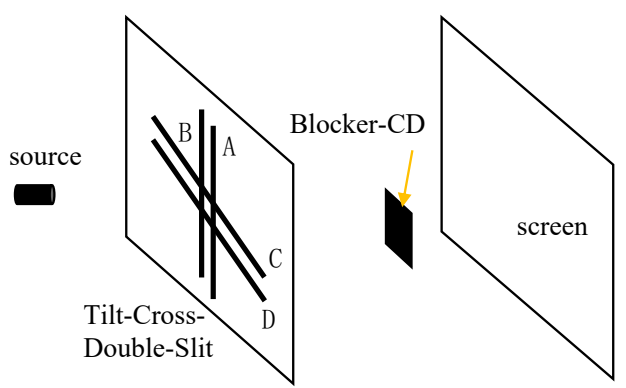

(a)

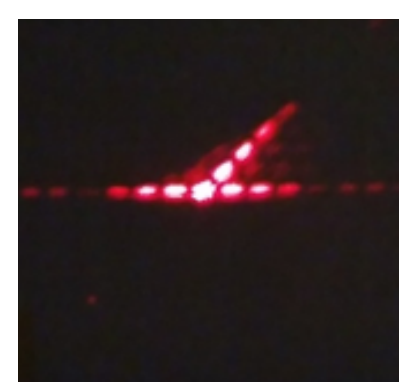

(b)

Figure 3.4-2 Tilt cross-double slit experiment with blocker-CD

Observation (Figure 3.4-2b): the tilt bottom-half fringes are blocked. The remaining fringes are not affected by blocking.

Experimental setup-3 (Figure 3.4-3a): Blocker-AB and blocker-CD were placed such that half of the interference pattern created by double slit-AB was blocked, simultaneously, half of the interference pattern created by double slit-CD was blocked. 


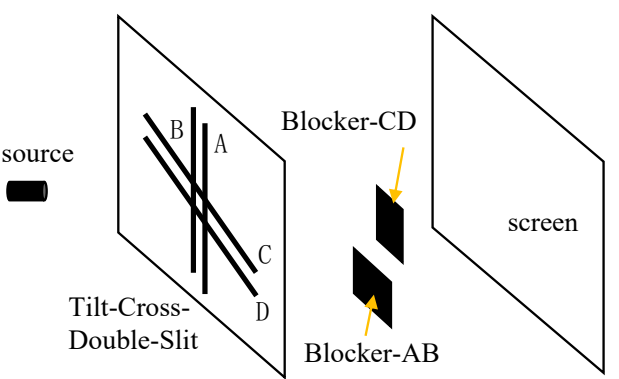

(a)

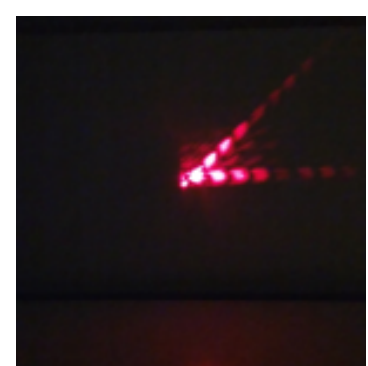

(b)

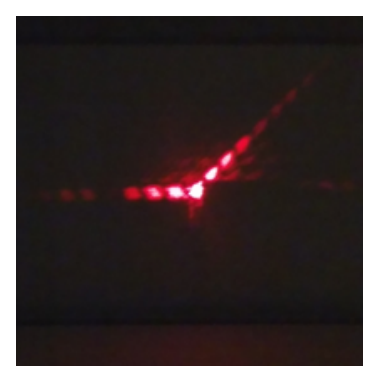

(c)

Figure 3.4-3 Tilt cross-double slit experiment with blocker-AB and blocker-CD

Observation: Figure 3.4-3b shows that the horizontal left half fringes are blocked, while the tilt bottom half fringes are blocked. The remaining fringes are not affected by blocking. Figure 3.4-3-c shows that the horizontal right half fringes are blocked, while the tilt bottom half fringes are blocked. The rest fringes are not affected by blocking. The observations show that photons move along trajectories.

Note that all experiments in Section 3.2 are observed by the naked eye, and there are no noticeable changes in the brightness of fringes. We cannot determine whether the trajectories cross.

We have shown that the $2 \mathrm{D}$ interference patterns are created independently and partially and that in Zone-3, photons move along trajectories and behave as particles.

\subsection{Which-Way-Cross-Double-Slit Experiments Supporting Trajectory Theory: Far Field}

The Which-way-Double-Slit experiments show that the motion of the particles/photons depends on whether both slits are open and would be different if one slit was closed. With only one slit open, the distribution of the photons on the screen would create a different pattern that shows the particle nature, according to the practical definition of wave/particle. Bohm's theory has same statement [17].

The observation of the regular Which-way-Double-Slit experiment is shown in Figure 3F, where an "observer" is set behind slit A (denoted by dashed slit A).

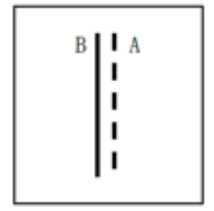

(a)

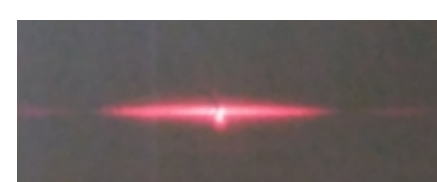

(b)

Figure 3F Regular Which-way-Double-Slit experiment

Figure $3 \mathrm{G}$ shows that half of the pattern is blocked.

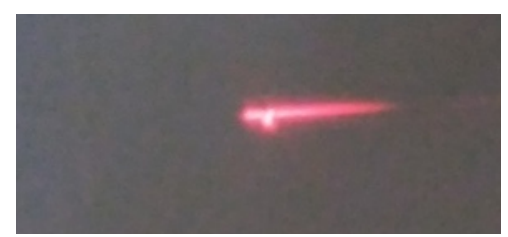

Figure 3G Which-way-Double-Slit experiment: blocking half pattern 
For Young's double slit experiment, if trajectory theory holds, e.g., trajectories do not cross, one knows which-way a photon passes through without observing and destroying the interference pattern. A which-way 2D cross-double slit experiment was performed (Figure 3H).



(a)

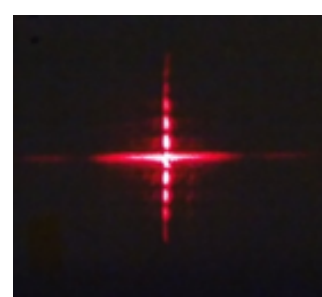

(b)

Figure 3H Which-way 2D cross-double slit experiment

By the same argument that the which-way 1D double slit experiments support the complementarity principle, which-way-2D-cross-double slit experiments oppose the Bohr's complementarity principle. Namely, in the same experiment with the same light source, the same diaphragm of the cross-double slit and the same detector, light/photons behave as both waves and particles.

Now let us block different portions of the patterns of the "which-way experiments".

Experiment-3.5: Which-way-Cross-Double-Slit experiments with blocker(s)

Experimental Setup-1 (Figure 3.5-1a): Place blocker-AB at different positions.

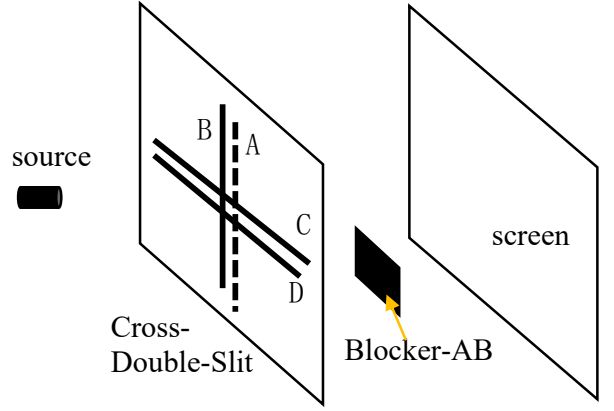

(a)

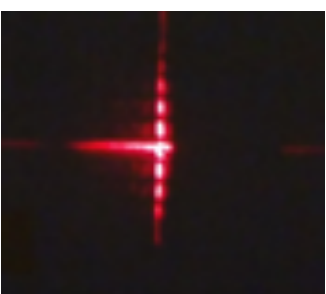

(b)

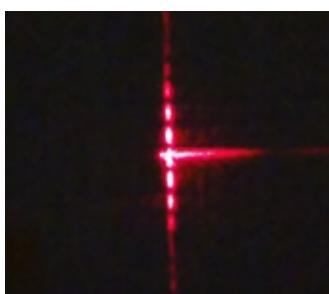

(c)

Figure 3.5-1 Which-way 2D cross-double slit experiments with blocker-AB

Observations: Figure 5.5-1b shows that block-AB blocks the right half of the pattern. Figure 3.5-1c shows that the left half of the pattern created by double slit-AB is blocked. In both setups, the remaining parts of both the pattern and the interference pattern are not affected, which is the consequence of photons propagating along trajectories.

Experimental Setup-2 (Figure 3.5-2a): Place blocker-CD between the cross-double slit and the screen. 


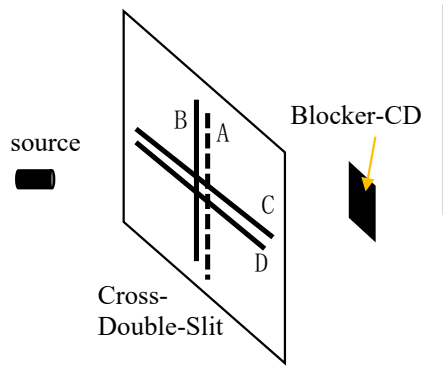

(a)

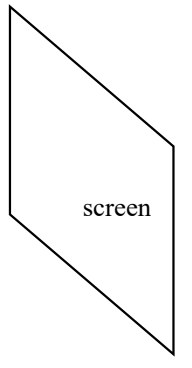

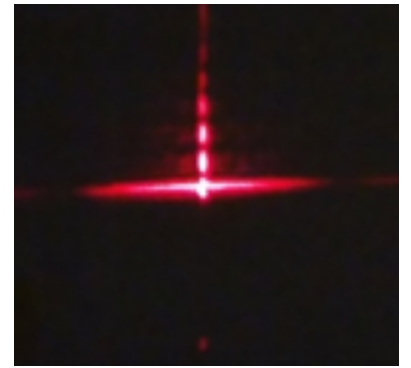

(b)

Figure 3.5-2 Which-way 2D cross-double slit experiments with blocker-CD

Observations: Figure 3.5-2b shows that block-CD blocks the bottom half of the interference pattern created by the double slit-CD, but it does not affect the top half of the interference pattern. The pattern created by the double slit-AB is not affected.

Experimental Setup-3 (Figure 3.5-3a): Placing both blocker-AB and blocker-CD between the cross-double slit and the screen.

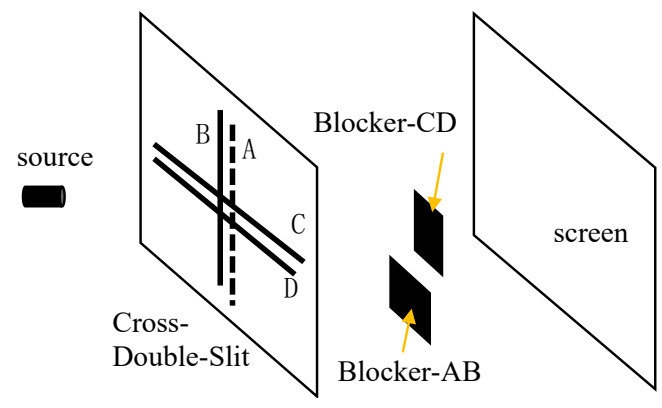

(a)

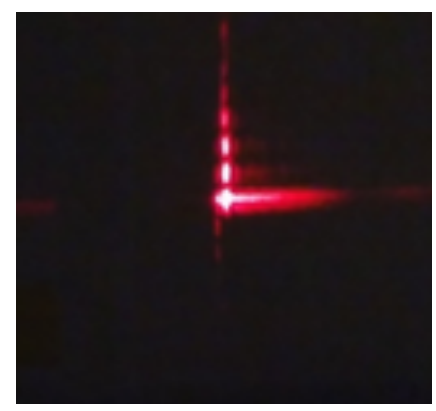

(b)

Figure 3.5-3 Which-way 2D cross-double slit experiments with blocker-AB and blocker-CD

Observations: Figure $3.5-3 \mathrm{~b}$ shows that block-CD blocks the bottom half of the interference pattern created by the double slit- $\mathrm{CD}$, but does not affect either the top half of the interference pattern or the right half of the pattern created by the double slit-AB. Block-AB blocks the left-half pattern, but does not affect both the right half of the pattern and the top half of the interference pattern. The phenomena are the consequences of photons propagating along trajectories.

Which-way 2D tilt cross-double slit experiments have been performed.

Next let us perform the which-way tilt cross-double slit experiments with blocker(s). 


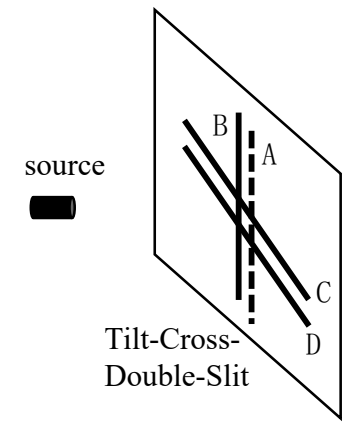

(a)

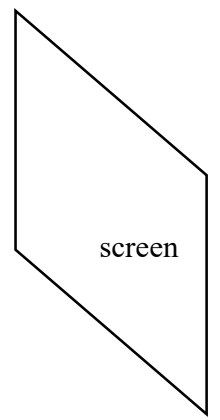

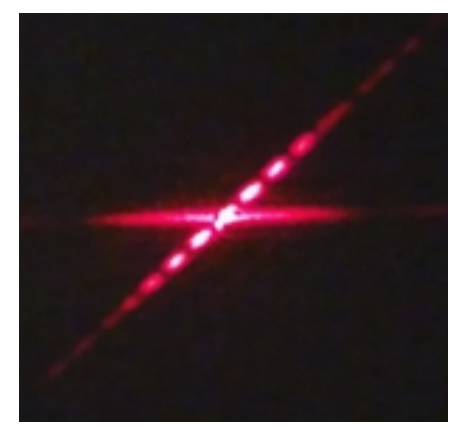

(b)

Figure 3I Which-way tilt cross-double slit experiments

Experiment-3.6: Which-way 2D tilt cross-double slit experiments with blocker(s). The experiment is performed in three setups.

Experimental Setup-1 (Figure 3.6-1a): Placing blocker-AB between the tilt cross-double slit and the screen.

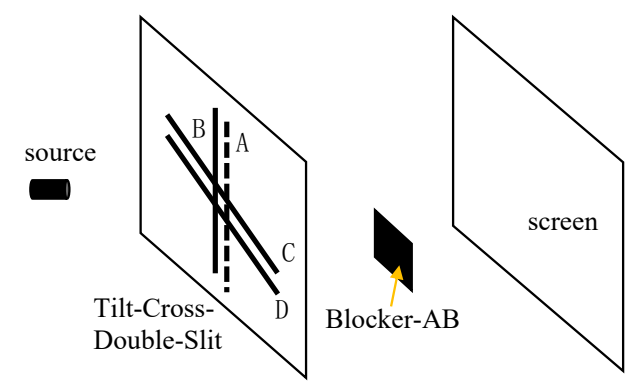

(a)

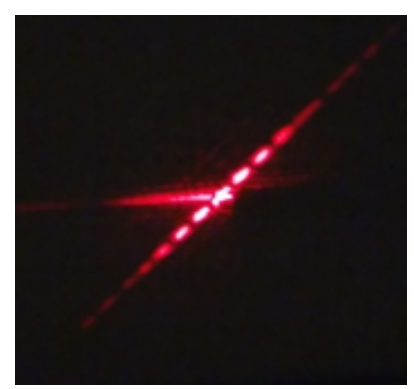

(b)

Figure 3.6-1 Which-way tilt cross-double slit experiment with blocker-AB

Observations: Figure 3.6-1b shows that block-AB blocks the left half of the pattern created by the double slit-AB with "observation at slit A", while it does not affect both the right half of the pattern and the interference pattern created by the double slit-CD.

Experimental Setup-2 (Figure 3.6-2a): Placing blocker-CD between the tilt cross-double slit and the screen.

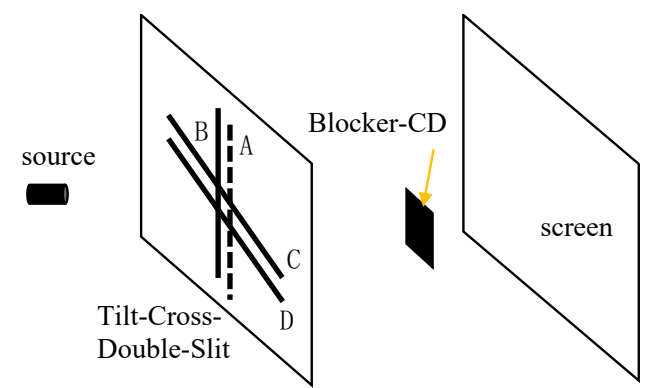

(a)

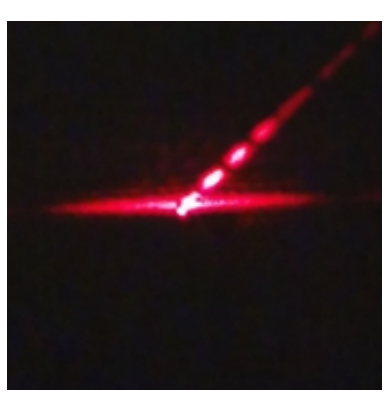

(b)

Figure 3.6-2 Which-way tilt cross-double slit experiment with blocker-CD 
Observations: Figure 3.6-2b shows that block-CD blocks the bottom half of the interference pattern created by the tilt double slit-CD, while it does not affect both the top half of the interference pattern and the pattern created by the double slit-AB.

Experimental Setup-3 (Figure 3.6-3a): Place both blocker-AB and blocker-CD between the tilt cross-double slit and the screen.

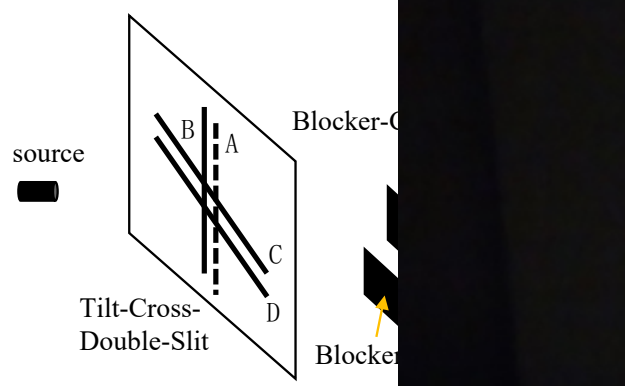

(a)

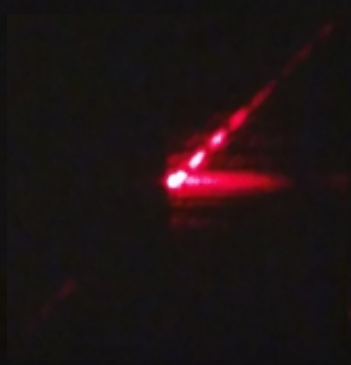

(b)

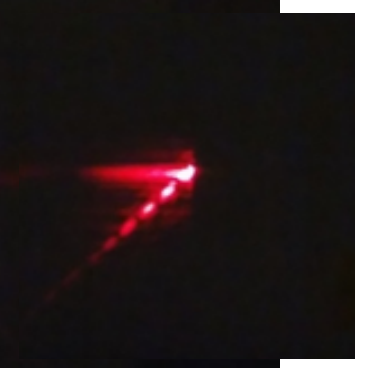

(c)

Figure 3.6-3 Which-way tilt cross-double slit experiment with blocker-AB and blocker-CD

Observations: Figure 3.6-3b shows that block-CD blocks the bottom half of the interference pattern created by the tilt double slit-CD, but it does not affect both the top half of the interference pattern and the right half of the pattern created by the double slit-AB. Block-AB blocks the left half of the pattern created by the double slit-AB with "observation at slit A", but it does not affect both the right half of the pattern and the top half of the interference pattern created by the tilt double slit-CD.

Figure 3.6-3c shows that block-CD blocks the top half of the interference pattern created by the tilt double slit-CD, but it does not affect both the bottom half of the interference pattern and the left half of the pattern created by the double slit-AB. Block-AB blocks the right half of the pattern created by the double slit-AB, but it does not affect both the left half of the pattern and the bottom half of the interference pattern created by the tilt double slit-CD.

Although each photon travels along its own trajectory, it is challenge to interpret the co-existence of wave-distribution and particle nature of the which-way-cross-double slit experiments.

\section{Discussion and Conclusion}

We have extended:

(1) the 1D-double slit experiments, which is the mystery of the quantum mechanics (Feynman), to

2D-cross-double slit experiments, which is more mysterious than the double slit experiments; and

(2) the which-way-1D-double slit experiments, which support the Bohr's complementarity principle, to the which-way-2D-cross-double slit experiments, which show the co-existence of the particle nature and the wave distribution and thus, violate the complementarity principle. It is challenge to interpret 
the cross-double slit experiment. A photon needs to know which slit it passes through and the angle of that slit relative to the certain direction to behave accordingly.

The standard wave interpretation of the double slit experiments states that the light beam behaves as waves from the time it is emitted until the time it lands on the screen. When it lands on the screen, the wave function collapse. To test the wave interpretation, we propose Postulate-1 and Postulate-2, which state that the photons emitted by a laser source behave as particles before passing through the double slit/cross-double slit (i.e., in Z-1), and before landing on the detector/screen (i.e., in Z-3).

The varieties of the comprehensive-double slit/cross-double slit experiments are performed. Indeed, those comprehensive experiments support Postulate-1 and -2.

The experiments in this article provide the information for theoretical development. The challenge is to find the mechanism of the wave distribution of photons as particles.

\section{Appendix:}

\section{A1: Novel Diaphragm of Double Slit for Testing Trajectory Theory}

In preliminary Experiment-3.1 and Experiment-3.2 of Section 3.1, the shields contact the diaphragms of the double slit (Figure 3.1-1 and 3.2-1). However, the "contact" is a macroscopic-type contact, i.e., actually, there are "gaps" between the shield and the diaphragm of the double slit. For further double slit experiments, we design new apparatuses to eliminate the gap between the shield and the double slit, they are one piece now (Figure A1).

$\mathrm{W}$

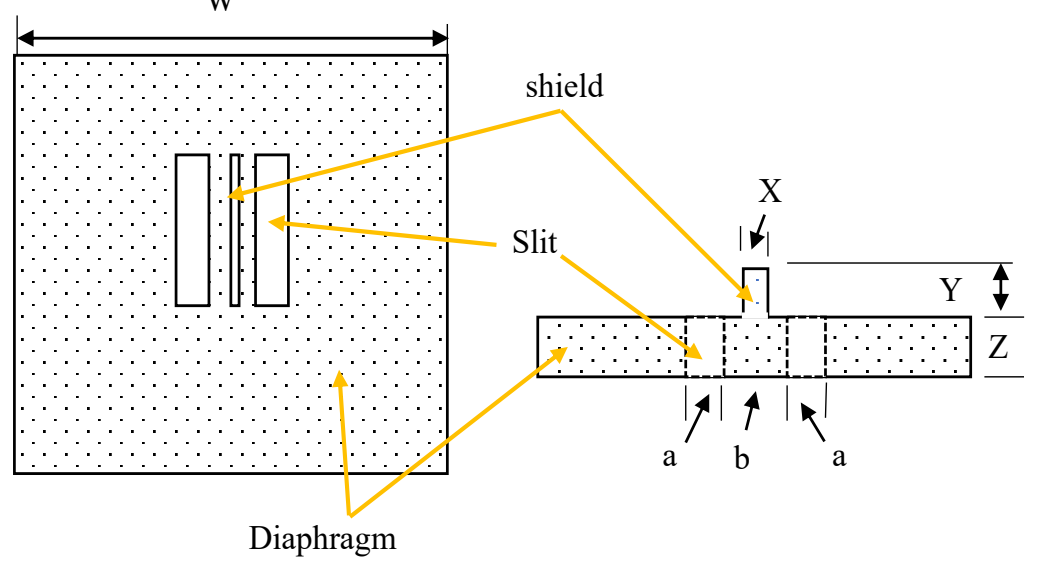

Figure A1 Novel Diaphragm for Double Slit Experiments

\section{A2. 2D-Cross-Double Slit Experiments}

\section{A2-1. 2D-Cross-double Slit}

To study further the double slit experiments and wave-particle duality, the cross-double slit experiments have been proposed/performed. The diaphragms (Figure A2.1) contain double slit crossing 
double slit, double slit crossing triple-slit, single-slit crossing double slit, single slit crossing triple-slit, hereafter, denoted all as "cross-double slit".
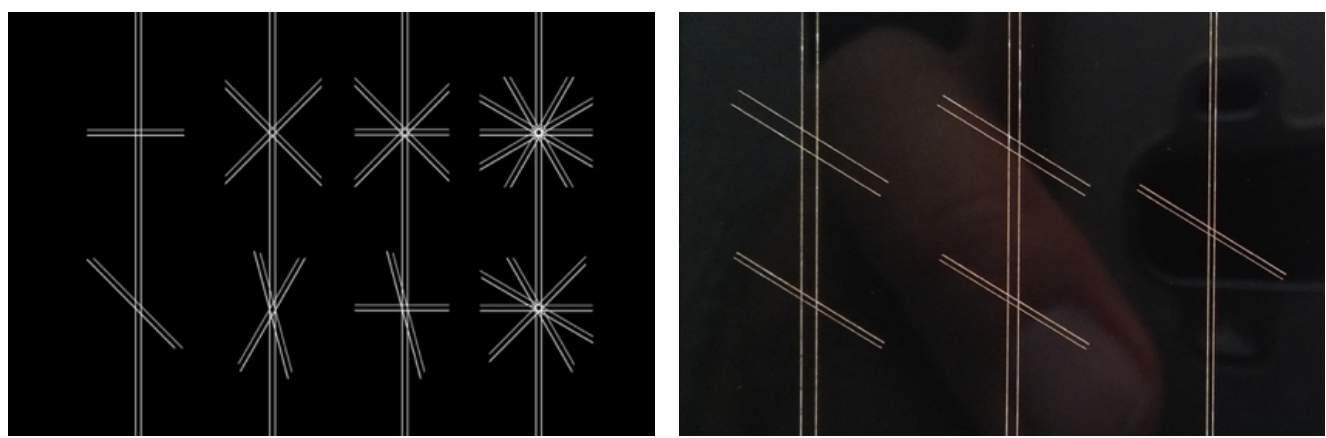

(a) Double slit crossing double slit: same/different spacing
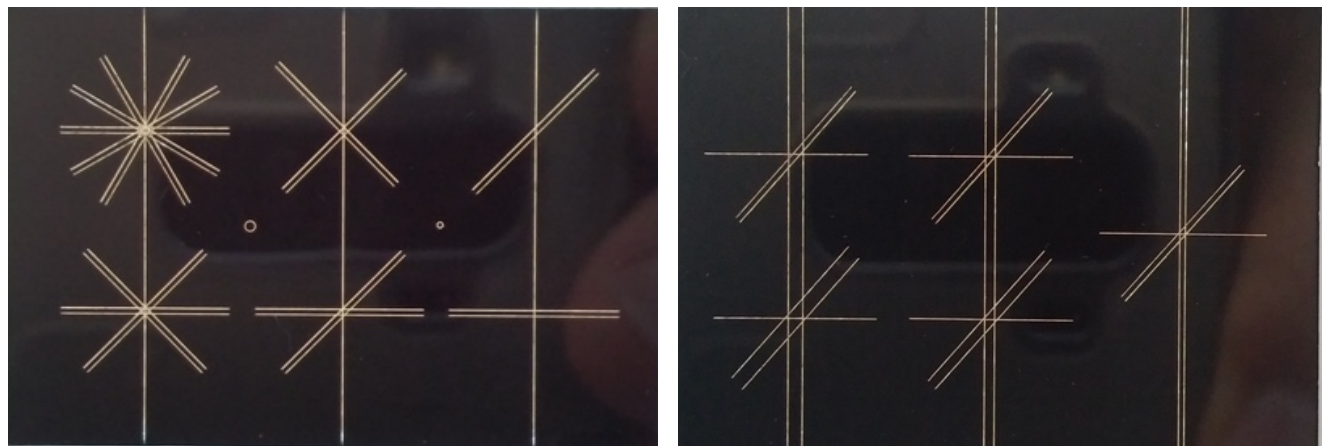

(b) Single slit crossing double slit: same/different spacing
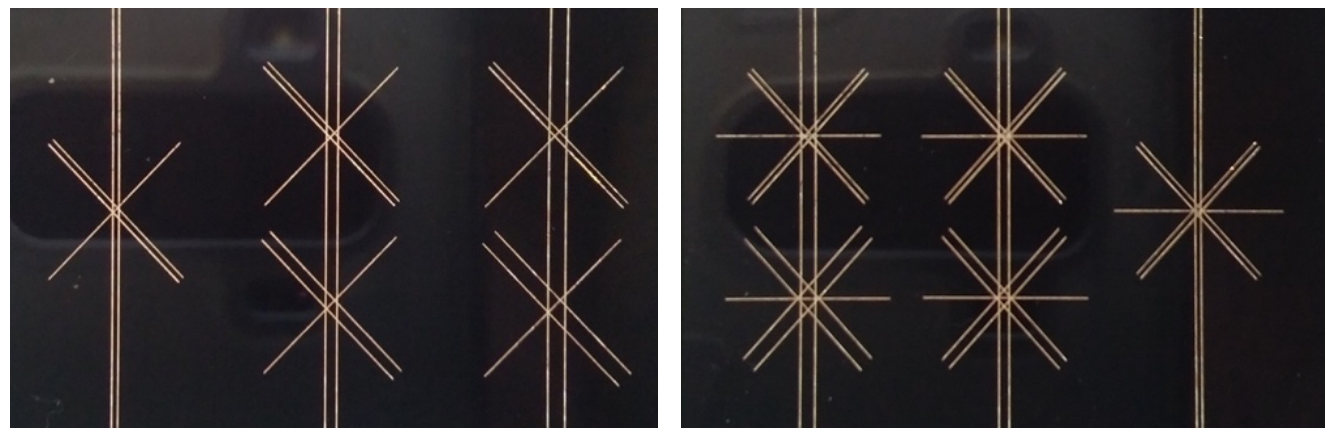

(c) Single slit crossing double slit: same/different spacing
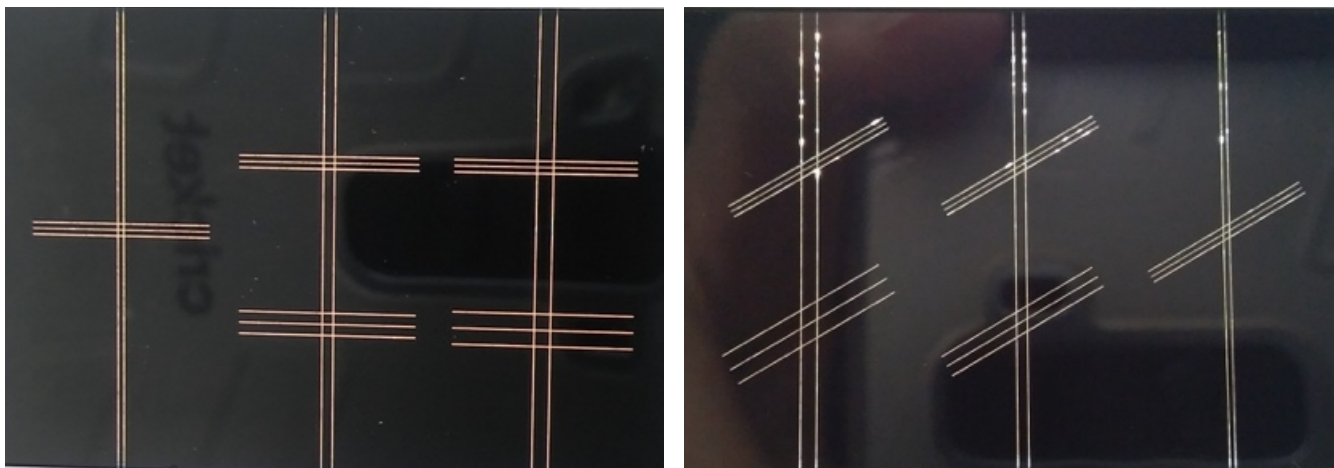

(d) Double slit crossing triple slit: same/different spacing 

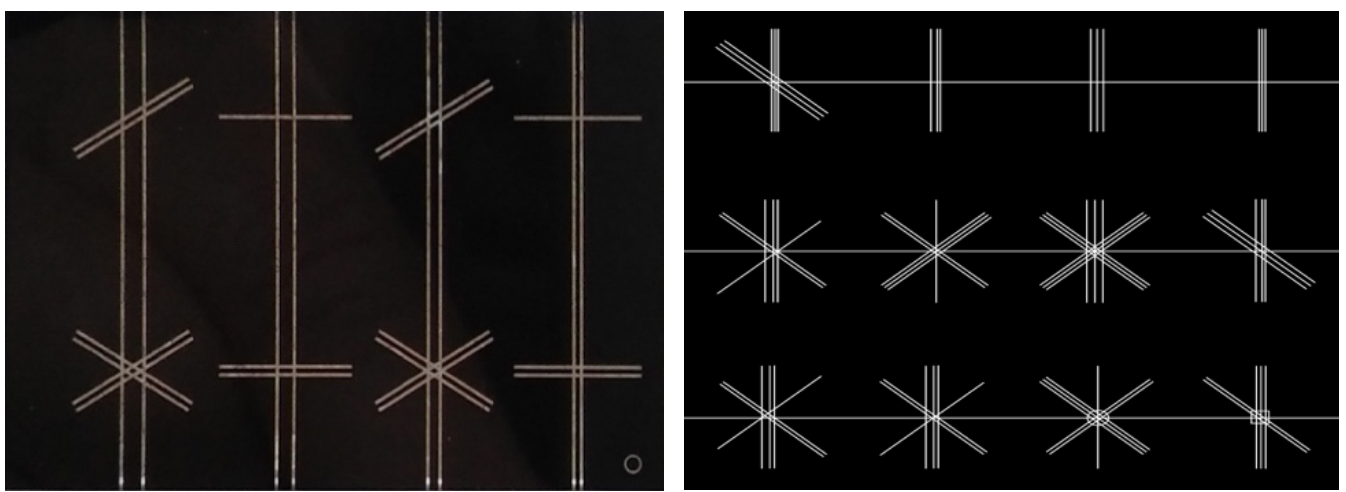

(e) Single slit/double slit crossing double slit/triple slit

Fig. A2-A Diaphragms of cross-double slit

\section{A2-2. Cross-Double Slit Experiments}

\section{A2-2-1. Double Slit Crossing Double Slit Experiments: Same Spacing}

The experimental setup of the cross-double slit experiments consists of a laser source, a slide/diaphragm of cross-double slit and a detector/screen. In the following experiments, we show the varieties of the cross-double slit and their patterns, which provide comprehensive data for building a theoretical model. To clearly show the evolution from the double slit experiments to cross-double slit experiments to comprehensive double slit/cross-double slit experiments, let us start with Young's double slit, then show the cross-double slit experiments and the comprehensive double slit/cross-double slit experiments.
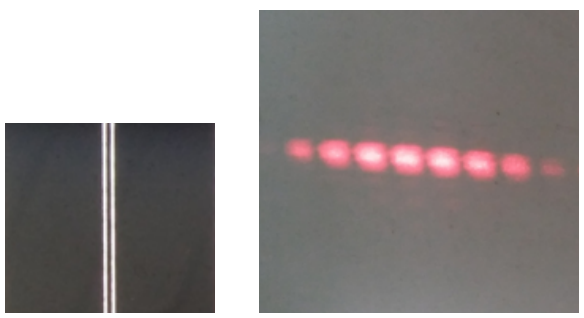

Figure A2-B. Young's double slit experiment

Experiment-A2.1 (Figure A2.1): Double slit crossing double slit at the right angle and its pattern.
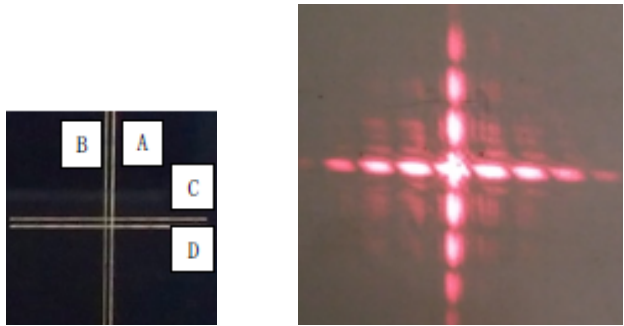

Figure A2.1
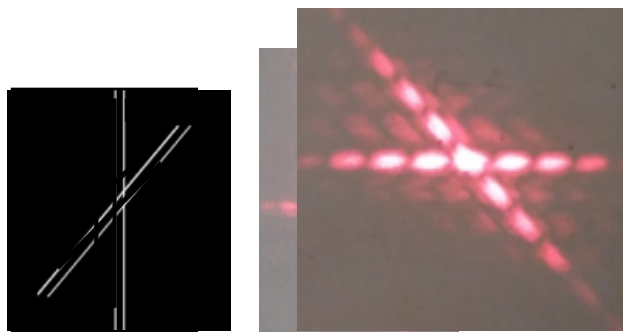

Figure A2.2

Experiment-A2.2 (Figure A2.2): The tilt double slit crossing the vertical double slit and its pattern. 
Experiment-A2.3 (Figure A2.3): Three double slits crossing at the same spot and its pattern. The angle between the tilt double slit and the vertical double slit is $45^{\circ}$.

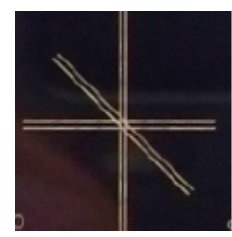

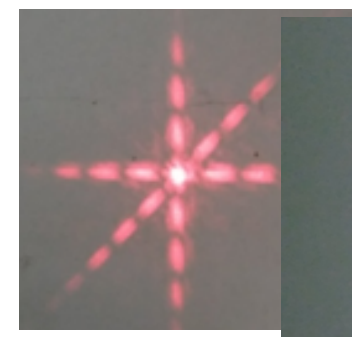

Figure A2.3
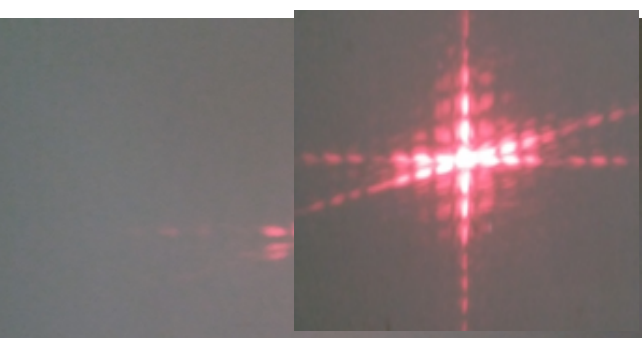

Figure A2.4

Experiment-A2.4 (Figure A2.4): Three double slits crossing at the same spot and its pattern. The angle between the tilt double slit and the vertical double slit is $15^{\circ}$.

Experiment-A2.5 (Figure A2.5): Three double slits crossing and its pattern.
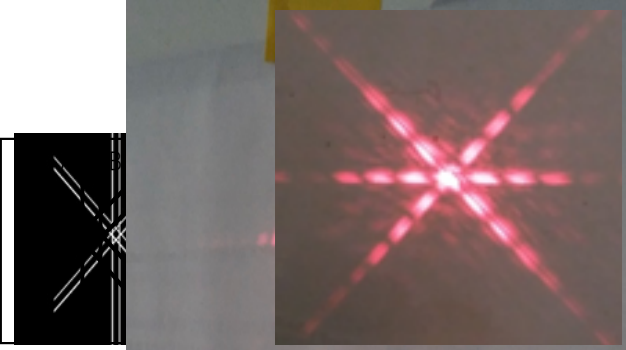

Figure A2.5
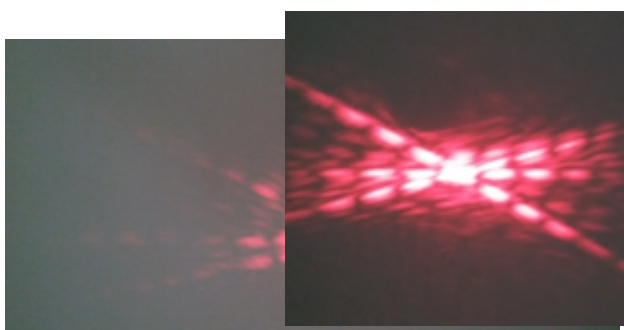

Figure A2.6

Experiment-A2.6 (Figure A2.6): Three crossing double slits and its pattern. The tilt angles are $15^{0}$ and $30^{0}$ respectively.

Experiment-A2.7 (Figure A2.7): Four crossing double slits and its pattern.

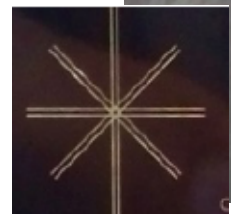



Figure A2.7
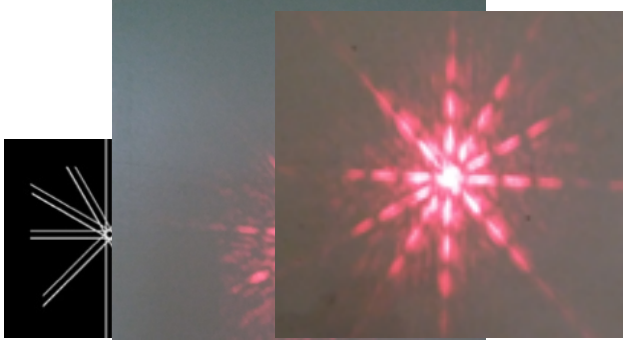

Figure A2.8

Experiment-A2.8 (Figure A2.8): Five crossing double slits and its pattern.

Experiment-A2.9 (Figure A2.9): Six crossing double slits and its pattern.
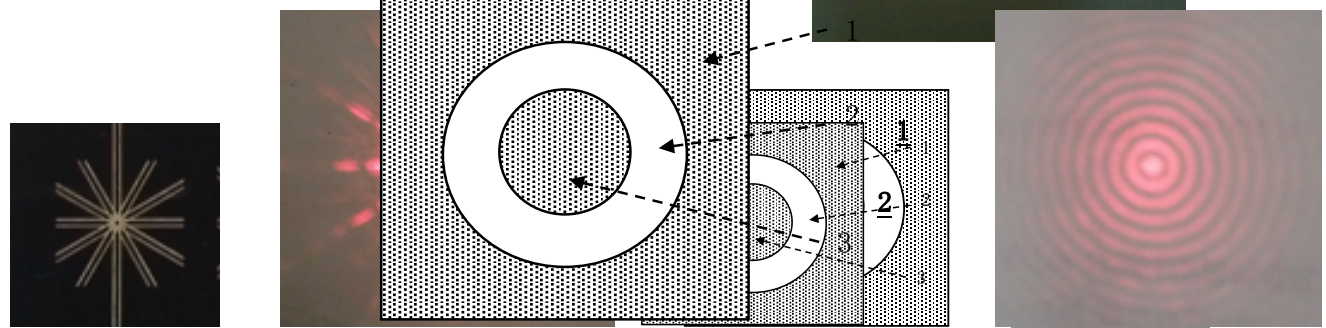
Experiment-A2.10 (Figure A2.10): Rotation Invariance of double slit/cross-double slit.

The combination of Figures A2.7, A2.8 and A2.9 suggests that the double slit and cross-double slit have rotation invariance around their normal vectors. If we increase the number of double slits that intersect at the same spot, the shape of the intersection will approach a circular disc. When rotating either a double slit or a cross-double slit around its normal vector, each slit is tangent to the intersection and forms disc-3, which is surrounded by ring-2. The final pattern can be obtained by either rotating the double slit/cross-double slit apparatus during the experiment or by applying an apparatus of disc-3 of the diameter that is equal to the spacing between two slits, and ring- 2 of the width that is equal to the width of a single slit.

We argue that Figure A2.10 indicates that the double slit and the cross-double slit have rotation-invariance around their normal vector.

\section{A2-2-2. Double Slit Crossing Double Slit Experiments: Different Spacing}

Experiment-A2.11 (Figure A2.11). Double slit crossing double slit of different spacing (the left of Figure A2.11) and its pattern (the right of Figure A2.11).
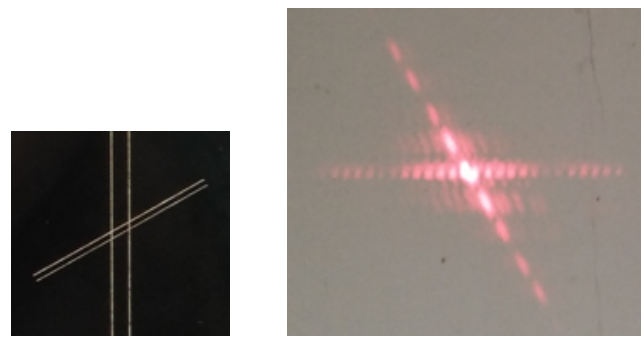

Figure A2.11

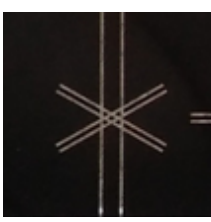

Figure A2.12

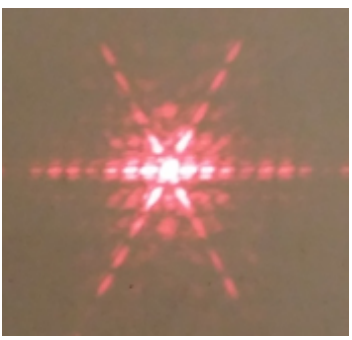

Experiment-A2.12 (Figure A2.12): Double slit crossing double slit of different spacing and its pattern.

Conclusion: It is a challenge to consistently interpret the experiments in Section A2.1.

\section{A2-3. Double slit Crossing Triple-Slit Experiments}

A typical triple slit and its pattern is shown in Figure A2.
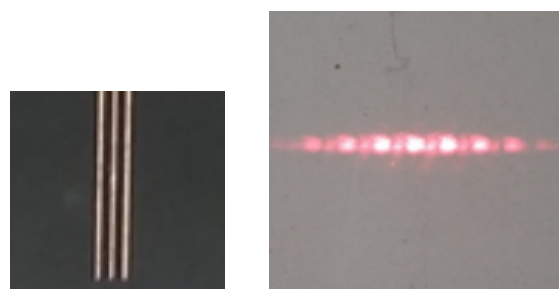

Figure A2-C. Triple Slit and its pattern

\section{A2-3-1. Double slit Crossing Triple-Slit Experiments: Same Spacing}


Experiment-A2.13 (Figure A2.13): Double slit crossing triple slit and its pattern. All of spacing between slits are $0.25 \mathrm{~mm}$.
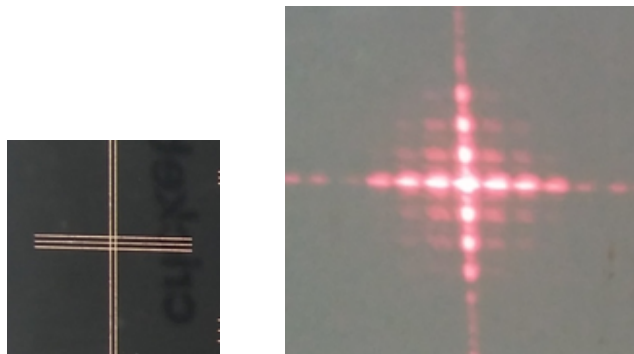

Figure A2.13
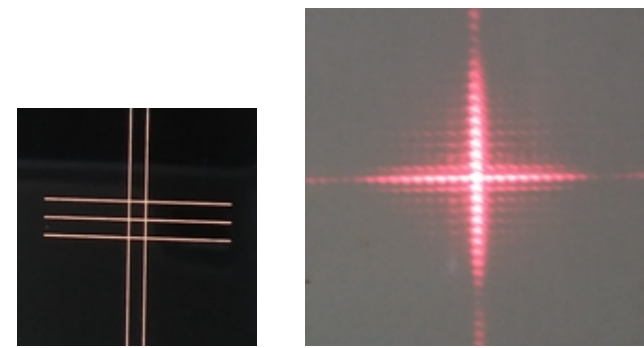

Figure A2.14

Experiment-A2.14 (Figure A2.14): Double slit crossing triple slit. Spacings between slits are 0.75 mm.

Experiment-A2.15 (Figure A2.15): Double slit crossing tilt triple slit. Spacings between slits are 0.25 $\mathrm{mm}$.
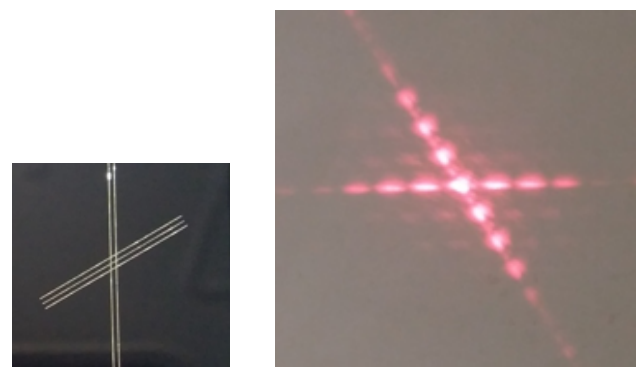

Figure A2.15

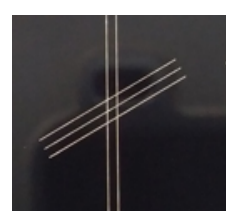

Figure A2.16

Experiment-A2.16 (Figure A2.16): Double slit crossing tilt triple slit. Spacings between slits are 0.5 $\mathrm{mm}$.

\section{A2-3-2. Double slit Crossing Triple-Slit Experiments: Different Spacing.}

Experiment-A2.17 (Figure A2.17): The spacing between two vertical slits is $0.5 \mathrm{~mm}$, and the spacing between three horizontal slits is $0.25 \mathrm{~mm}$.

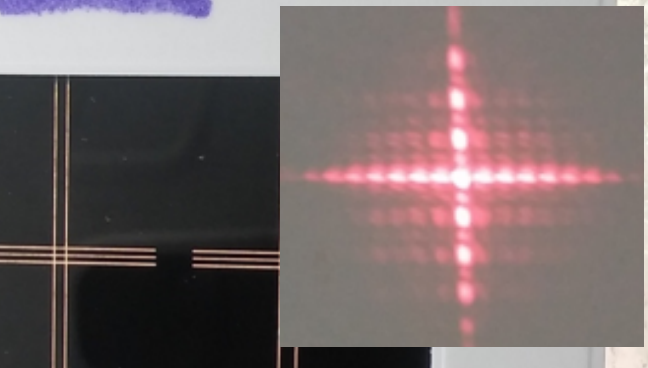

Figure A2.17
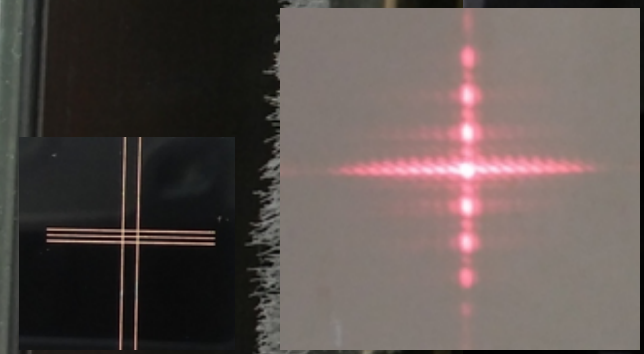

Figure A2.18

Experiment-A2.18 (Figure A2.18): The spacing between two vertical slits is $0.75 \mathrm{~mm}$, and the spacing between three horizontal slits is $0.25 \mathrm{~mm}$. 
Experiment-A2.19 (Figure A2.19): The spacing between two vertical slits is $0.5 \mathrm{~mm}$, and the spacing between two of three tilt slits is $0.25 \mathrm{~mm}$.
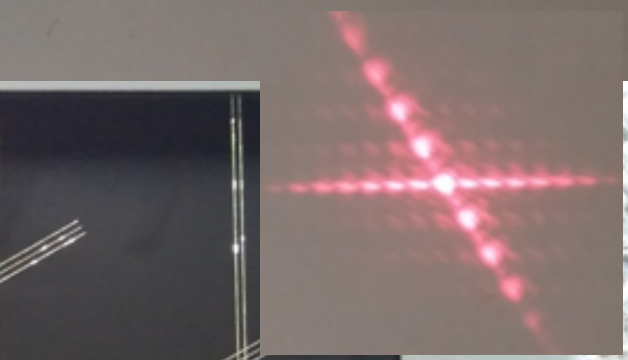

Figure A2.19

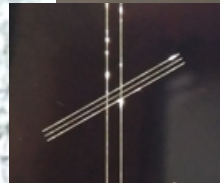

Figure A2.20

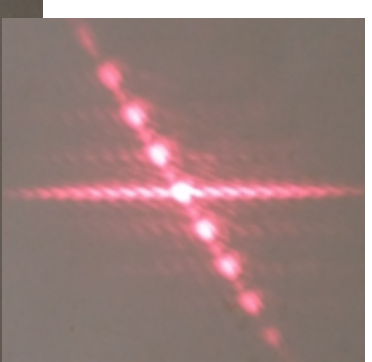

Experiment-A2.20 (Figure A2.20): The spacing between two vertical slits is $0.75 \mathrm{~mm}$, and the spacing between three tilt slits is $0.25 \mathrm{~mm}$.

Conclusion: It is a challenge to consistently interpret the experiments in Section A2.

\section{A3. Which-Way-Cross-Double-Slit Experiments}

\section{A3-1. Which-Way-Cross-Double-Slit Experiments: Single-Slit Crossing Double slit}

To explore the nature of photons further, 1D-double slit experiment was extended to which-way-1D-double-slit experiment. Technically feasible realizations of which-way experiments were proposed in the 1970s $[19,20]$. A photon is observed near a slit by utilizing a photoelectric detector to register the photon; practically it blocks the paths of photons, namely equivalent to covering a slit $[21,22]$. When the photon is detected, the interference pattern disappears. The varieties of the which-way-double-slit experiments have been proposed and performed at classical level and quantum level.

At the classical level, using a laser light source, the which-way-2D-cross-double slit experiments have been proposed (Figure A2D-a) and performed. At the quantum level, the double-double slit apparatus (two separate double slits) is utilized to perform the which-way-double-slit experiments with quantum entangled photons (Figure A2D-b) [8].

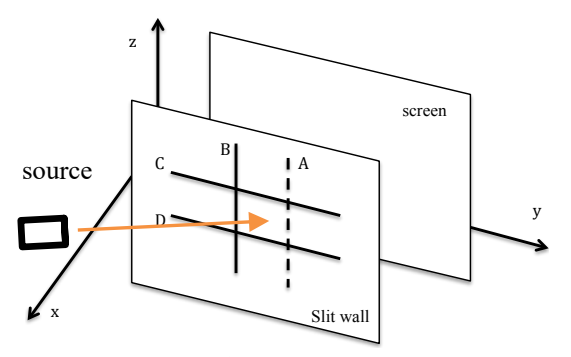

(a)

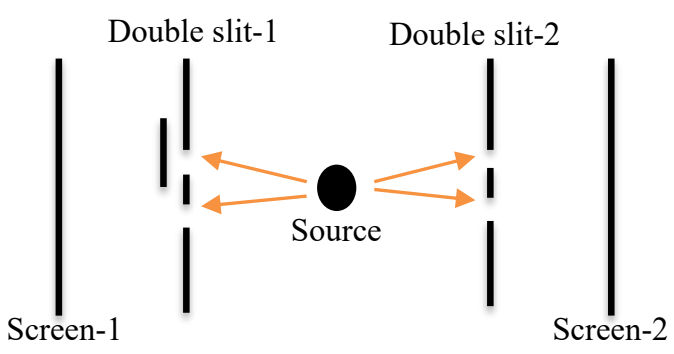

(b)

Figure A2D. Two kinds of which-way experiments 
Note that in both the classical level and quantum level, the cross-double slit and double-double slit are macroscopic devices. In each experiment, one light source is used.

We argue that, if we shift the focus from detecting photons at one slit to observe the final patterns (to see whether the pattern show the particle nature or wave distribution), then detecting a photon behind the slit of the double slit is equivalent to using a single slit, the logic chain is the following (Figure A2E):

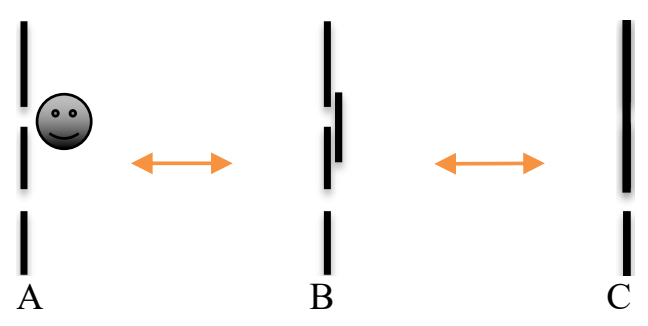

Figure A2E. Observing $(\mathrm{A})=$ blocking $(\mathrm{B})=\operatorname{single}$ slit $(\mathrm{C})$

A) To observe photons either behind or in front of a slit is equivalent to block photons either after or before passing through the slit;

B) To block photons after/before passing through the slit is equivalent to cover that slit;

C) To cover the slit is equivalent to using a single slit instead of using a double slit and covering one slit.

Thus, to perform which-way experiments with the diaphragm of the cross-double slit, we made one of the double slits as a single slit.

\section{A3-2. Which-Way-Cross-Double-Slit Experiments: Same Spacing}

Figure A2F shows the standard which-way-double slit experiment, where the left drawing is the double slit with an "observer" at slit "A", and the right is the pattern. Figure A2G shows the Which-way-Cross-Double-Slit.
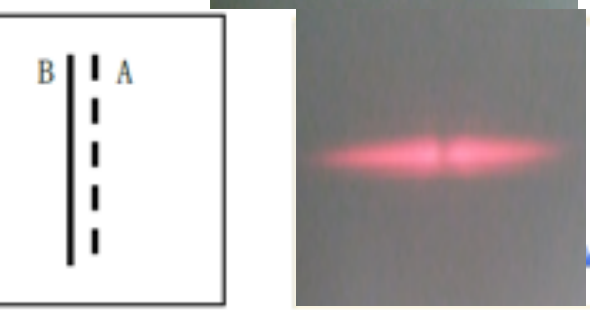

Figure A2F
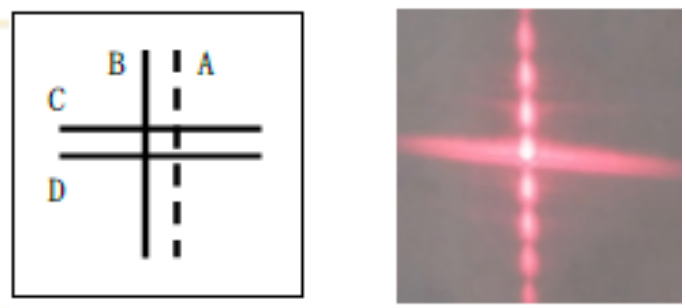

Figure A2G

The significant difference is that, for the standard which-way-double slit experiment, remaining photons as particles must go through slit "B", so it is confirm the wave-particle duality. For the which-way-cross-double-slit experiment, if photons do not go through slit "A", photons can go through 
either slit "B" or "C" or "D". A photon needs to know which slit it passing through to determine how to behave. If go through slits "C" and "D", photons need to behave as waves. If go through slit "B", photons need to behave as waves, namely, particle nature and wave distribution emerge in the same experiment.

Experiment-A2.21 (Figure A2.21): Single-slit crossing double slit.

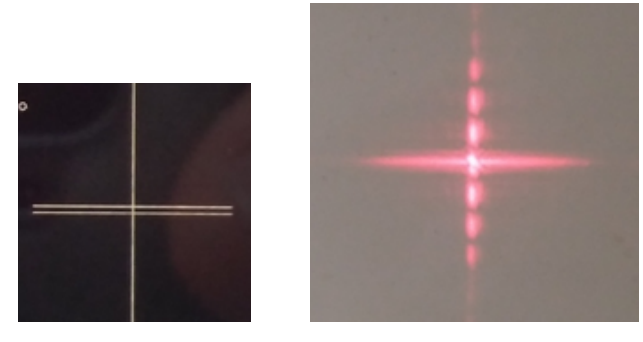

Figure A2.21
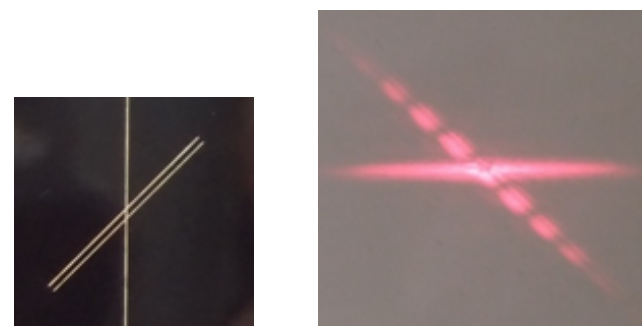

Figure A2.22

Experiment-A2.22 (Figure A2.22): Single-slit crossing tilt double slit

Experiment-A2.23 (Figure A2.23): Single slit crossing two double slits of the same spacing.
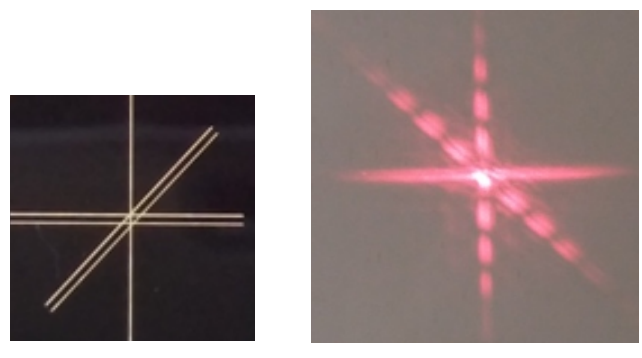

Figure A2.23

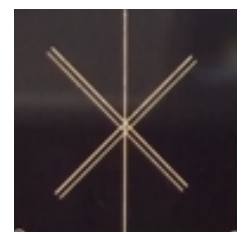

Figure A2.24

Experiment-A2.24 (Figure A2.24): Single slit crossing two tilt double slits of same spacing.

Experiment-A2.25 (Figure A2.25): Single slit crossing three double slits of same spacing.
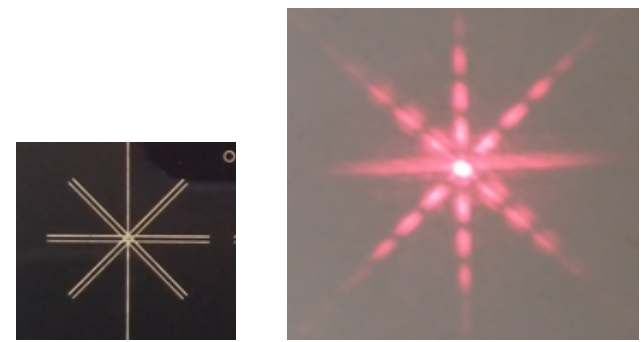

Figure A2.25
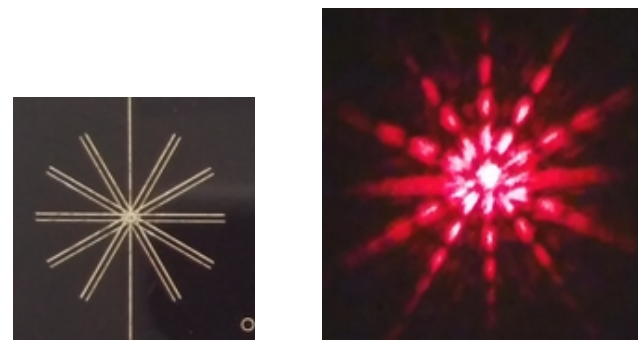

Figure A2.26

Experiment-A2.26 (Figure A2.26): Single slit crossing five double slits of the same spacing.

\section{A3-3. Which-Way-Cross-Double-Slit Experiments: Different Spacing}

Experiment-A2.27 (Figure A2.27): Single slit crossing two double slits of different spacings. 

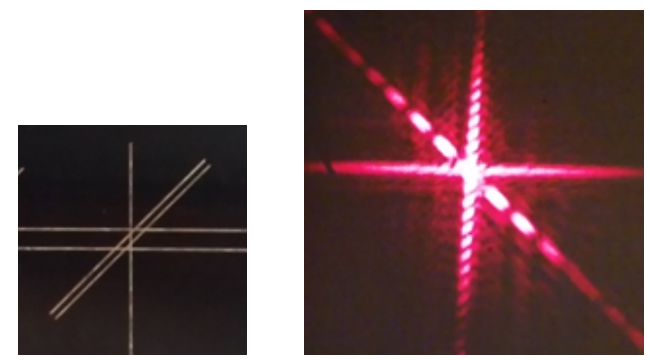

Figure A2.27
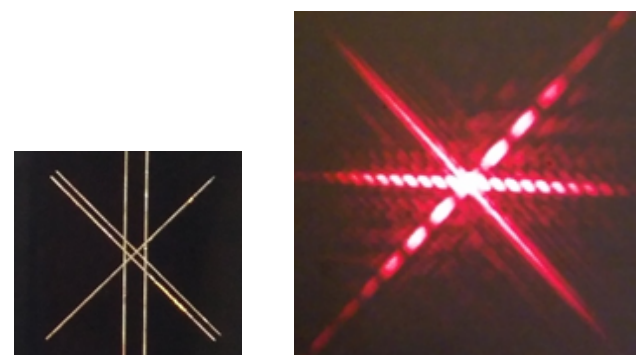

Figure A2.28

Experiment-A2.28 (Figure A2.28): Single slit crossing two double slits of different spacings.

Experiment-A2.29 (Figure A2.29): Single slit crossing three double slits of different spacings.
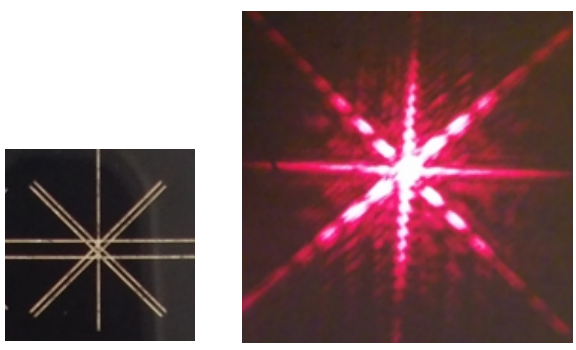

Figure A2.29

\section{A3-4. Which-Way-Cross-Double-Slit Experiments: Triple Slit}

Experiment-A2.30 (Figure A2.30): Single slit crossing triple slit (narrower spacing)

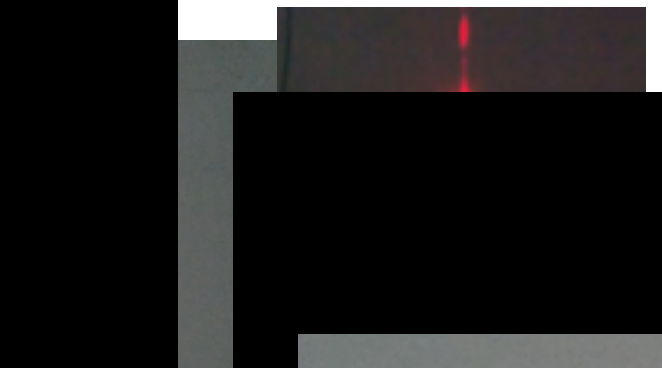

Figure A2.30

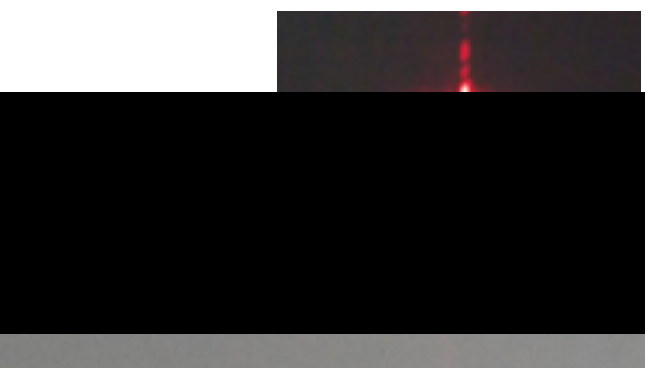

Figure A2.31

Experiment-A2.31 (Figure A2.31): Single slit crossing triple slit (wider spacing)

Experiment-A2.32 (Figure A2.32): Single slit crossing triple slit of nonuniform spacing.
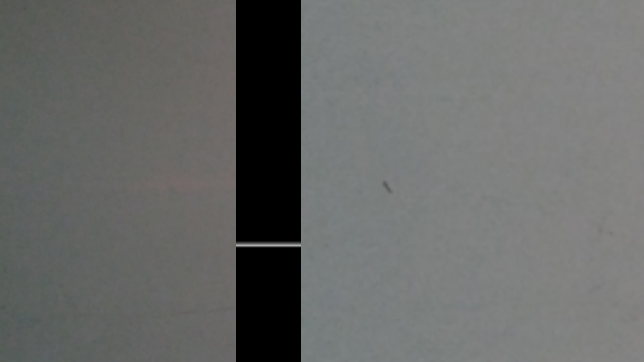

Figure A2.32
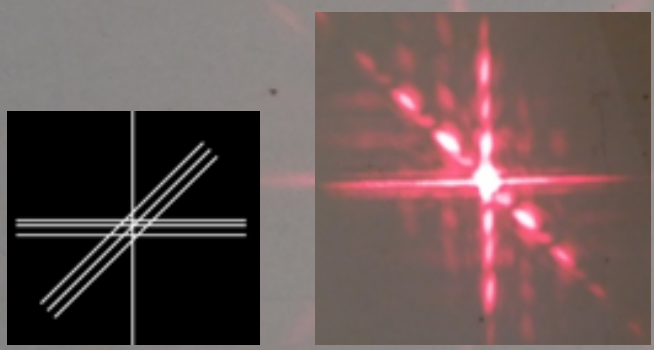

Figure A2.33

Experiment-A2.33 (Figure A2.33): Single slit crossing two triple slits of different/nonuniform spacing. 
Experiment-A2.34 (Figure A2.34): Two single slits crossing double slit and triple slit of different spacing.
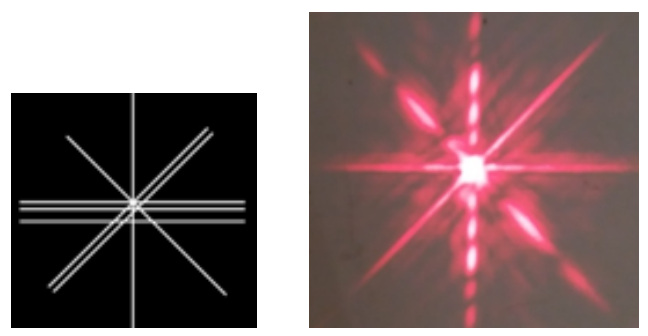

Figure A2.34
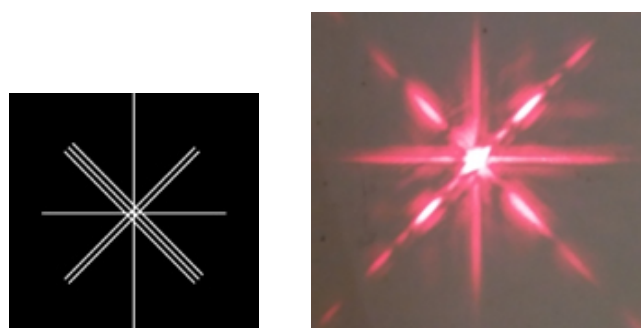

Figure A2.35

Experiment-A2.35 (Figure A2.35): Two single slits crossing double slit and triple slit of same spacing.

Conclusion: the which-way-cross-double-slit experiments show the co-existence of the particle nature and the wave-distribution, which violates Bohr's complementarity principle. It is challenge to interpret the experiments in Section 3.3 consistently.

\section{References}

1. A. Ananthaswamy, "Through Two Doors at Once”, Dutton, New York, NY, (2018).

2. A. Robinson, “The Last Man Who Knew Everything”. New York, NY: Pi Press., (2006).

3. R. Feynman, R. Leighton, and M. Sands, "The Feynman Lectures on Physics" (Addison-Wesley, Reading, 1966), Vol. 3.

4. S. Rashkovskiy, Is a rational explanation of wave-particle duality possible? arXiv 1302.6159 [quant-ph] 2013.

5. G. Greenstein and A.G. Zajonc, The Quantum Challenge: Modern Research on the Foundations of Quantum Mechanics, Jones and Bartlett, Boston, 1997.

6. J. Baggott, The Quantum Story: A History in. Oxford University Press, 2011.

7. R. Ionicioiu and D.R. Terno, Phys. Rev. Lett. 2011, 107, 230406.

8. Kaur, M., Singh, M. Quantum double-double-slit experiment with momentum entangled photons. Sci Rep. 2020, 10, 11427. https://doi.org/10.1038/s41598-020-68181-1.

9. Hui Peng, "Cross-Double slit Experiment and Extended-Mach-Zehnder Interferometer", dx.doi.org/10.7392/openaccess.45011872, 2019.

10. Hui Peng, "Observation of Cross-double slit Experiments”. International J. of Physics. 8(2), 39-41. DOI: 10.12691/ijp-8-2-1. 2020.

11. de Broglie, in "Ondes et mouvements" [Waves and Motions], Gauthier-Villars, Paris, 1926.

12. de Broglie, L. Interpretation of quantum mechanics by the double solution theory. Ann. Foundation Louis de Broglie, 12: no 4. 1987. 
13. Bohm, D. A Suggested Interpretation of the Quantum Theory in Terms of "Hidden" Variables. I, Phys Rev., 85: 166 - 193. 1952.

14. C. Philippidis, C. Dewdney and B.J. Hiley, "Quantum Interference and the Quantum Potential", Il Nuovo Cimento 1979, vol. 52B, No.1.

15. P.R.Holland, The Quantum Theory of Motion, An account of the de Broglie-Bohm causal interpretation of quantum mechanics, Cambridge University Press 1993.

16. S. Goldstein, “Bohmian Mechanics”, Plato.Stanford.edu, 2017.

17. R. Tumulka, "Bohmian Mechanics", arXiv: 1704.08017v2 [quant-ph] April 2018.

18. Bartell, L. "Complementarity in the double slit experiment: On simple realizable systems for observing intermediate particle-wave behavior". Physical Review D, 21 (6): 1698-1699. 1980.

19. Zeilinger, A. "Experiment and the foundations of quantum physics". Reviews of Modern Physics, 71 (2): S288 S297. 1999.

20. S. Frabboni, G. Gazzadi, and G. Pozzi, Appl. Phys. L. 2010, 97, 263101.

21. H. J. W. Müller-Kirsten, Introduction to Quantum Mechanics. World Scientific 2006, 14. 